Comparative Population Studies

Selected Articles in German Vol. 40 (2015): 87-114

(Erstveröffentlichung: 20.03.2015)

\title{
Soziale Unterschiede in der Säuglingssterblichkeit in Rostock im 19. Jahrhundert
}

\author{
Eine demografische Analyse anhand von Kirchenbuchdaten
}

\author{
Michael Mühlichen, Rembrandt D. Scholz, Gabriele Doblhammer
}

Zusammenfassung: Der Beitrag untersucht die historische Entwicklung der Säuglingssterblichkeit in der Hansestadt Rostock und widmet sich im Speziellen der Frage, inwieweit sozioökonomische Faktoren die Höhe der Säuglingssterblichkeit im frühen 19. Jahrhundert beeinflussten. Es lässt sich für die Stadt ein im deutschlandweiten Vergleich äußerst niedriges Säuglingssterblichkeitsniveau feststellen, besonders für das erste Drittel des Jahrhunderts. Dabei kann ein signifikanter Einfluss der beruflichen Schicht des Vaters auf die Überlebenschancen des Kindes im ersten Lebensjahr für das frühe 19. Jahrhundert nachgewiesen werden: Neugeborene von beruflich schlechter gestellten Vätern weisen ein größeres Sterberisiko im ersten Lebensjahr auf als die Nachkommen beruflich besser gestellter Väter. Als Datengrundlage dienen die Beerdigungs- und Taufregister der Rostocker Jakobikirche, welche weitgehend erhalten und zu einem großen Teil digitalisiert sind. Auf der Basis dieser Individualdaten wird erstmals ein Ereignisdatenanalysemodell im Zusammenhang mit der Säuglingssterblichkeit in einer deutschen Stadt im 19. Jahrhundert untersucht. Des Weiteren zeigt dieser Beitrag erstmals die Säuglingssterbewahrscheinlichkeit der Stadt Rostock für das gesamte 19. Jahrhundert nach Geschlecht und schließt damit in zweifacher Hinsicht eine Forschungslücke.

Schlagwörter: Säuglingssterblichkeit · Rostock · Historische Demografie • Kirchenbücher · Ereignisdatenanalyse

\section{$1 \quad$ Einleitung}

Die Untersuchung von Säuglingssterblichkeit ist ein wichtiger Bestandteil der Mortalitätsforschung. Ende des 19. Jahrhunderts starben in manchen deutschen Regionen über ein Drittel der Lebendgeborenen im ersten Lebensjahr (Würzburg 1887, 1888; Prinzing 1900). Damit bestimmte die Säuglingssterblichkeit zu einem wesentlichen Teil die Höhe der Gesamtsterblichkeit. Heute beträgt die Säuglingssterbeziffer 
in Deutschland laut Statistischem Bundesamt nur noch 3,3\%o (31.12.2013). Wegen des geringen Ausmaßes und der geringen Variation hat sie ihren Einfluss auf die Lebenserwartung weitgehend verloren. Der Anstieg der Lebenserwartung in der ersten Hälfte des 20. Jahrhunderts in Deutschland ist wesentlich auf den Rückgang der Säuglingssterblichkeit zurückzuführen und auch maßgeblich für die Einleitung des ersten demografischen Übergangs verantwortlich.

Über die Entwicklung und die Determinanten der Säuglingssterblichkeit im 19. Jahrhundert gibt es eine Vielzahl von Studien. Dennoch sind einige Regionen in Deutschland von der Forschung bislang nur unzureichend erfasst und nicht alle Einflussfaktoren und Ursachen der regionalen Unterschiede sind geklärt (Imhof 1981; Gehrmann 2000, 2011; Kloke 1997). Das liegt vor allem an dem Fokus des Interesses in der Forschung und unterschiedlichen Methoden und Definitionen. Während heute international vergleichbare Abgrenzungen von Totgeburt und Lebendgeburt ${ }^{1}$ existieren, trifft dies für die Vergangenheit nicht immer zweifelsfrei zu. Eine Reihe von Fragen sind ungeklärt: Welche Rolle spielt die Dokumentation von Sterbefällen und Geburten vor und nach der Taufe? Wie wirken sich unterschiedliche Bildung und Religion auf die Registrierung aus? Wann wird eine Totgeburt dokumentiert? Seit wann werden welche Merkmale im Zusammenhang mit Säuglingssterblichkeit erhoben?

Mecklenburg-Schwerin ist eine Region, die sich im 19. Jahrhundert durch besonders niedrige Säuglingssterblichkeit und hohe Lebenserwartung im Vergleich zu anderen Regionen in Deutschland auszeichnete (Dippe 1857). Die historische demografisch-statistische Datenlage ist für das Gebiet Mecklenburg-Schwerin sehr gut. Historische Bevölkerungsdaten sind bis ins 19. Jahrhundert zurück als Individualdaten in Form von Listen der Haushalte für mehrere Volkszählungen flächendeckend für die gesamte Region vorhanden. Ebenfalls sind die Kirchenbücher fast vollständig erhalten. Dadurch stehen Individualdaten zur Dokumentation der demografischen Ereignisse zur Verfügung. Diese Art von Daten für die Forschung zu nutzen, erfordert jedoch einen erheblichen Arbeitsaufwand, da die existierenden Quellen erst digitalisiert werden müssen. ${ }^{2}$

Informationsquellen zur Säuglingssterblichkeit im jeweiligen lokalen Kontext gibt es mit der Einführung von Kirchenbüchern. Analysen zur Mortalität auf Basis

1 Abgrenzung in Deutschland: Eine Lebendgeburt liegt vor, wenn mindestens eines von drei Lebenszeichen auftritt (Einsetzen der natürlichen Lungenatmung, Pulsieren der Nabelschnur oder Schlagen des Herzens). Wenn keines der Merkmale beobachtet werden konnte, das Geburtsgewicht aber mindestens $500 \mathrm{~g}$ beträgt, spricht man von einer Totgeburt. Wenn sich keines der Merkmale gezeigt hat und das Geburtsgewicht weniger als $500 \mathrm{~g}$ beträgt, handelt es sich um eine Fehlgeburt, die nicht registriert wird (Deutsche Verordnung zur Ausführung des Personenstandsgesetzes (Kapitel 5, § 31)).

2 Zu nennen ist für Mecklenburg-Schwerin die Aufnahme der Volkszählung 1819, einschließlich der Stadt Rostock und deren Analyse. Hierbei handelt es sich um das Pilotprojekt Mecklenburg in der demographischen Transition des 18. und 19. Jahrhunderts. Von Rostocker Historikern sind außerdem verschiedene Forschungsergebnisse zur städtischen Bevölkerungs- und Sozialgeschichte zu dem Beginn des 19. Jahrhunderts vorgelegt worden (Krüger/Kroll 1998; Krüger 1998, 2000, 2003; Manke 2000, 2005a/b). 
von Kirchenbüchern stammen u.a. von Halley (1693), Kundmann (1737), Süßmilch (1761), Blayo (1975), Wrigley/Schofield (1981), Schultz (1991), Gehrmann (2000, 2011), Johansson (2004, 2009) und Breschi et al. (2014), wobei Gehrmann neben Kirchenbüchern auch sämtliche anderen verfügbaren bevölkerungsstatistischen Quellen als Datenbasis heranzieht.

Flächendeckende statistische Aussagen für ganze Regionen können erst mit der Einführung der amtlichen Statistik gewonnen werden. Als erstes Land begann Schweden ab dem Jahr 1751 mit der Dokumentierung demografischer Ereignisse auf nationaler Ebene. Die anderen europäischen Länder folgten ab Mitte des 19. Jahrhunderts (Westergaard 1932; Hollingsworth 1969; Rödel 1990; Ehling 1996). Durch die Reichsgründung und die Bildung des Kaiserlichen Statistischen Amts werden vergleichbare Daten für Deutschland zur regionalen Säuglingssterblichkeit erstmals ab 1875 gewonnen (Westergaard 1932: 239). Die Entwicklung der Säuglingssterblichkeit für ausgewählte Länder zeigt Abbildung 1. In Skandinavien ist ein Rückgang der Säuglingssterblichkeit über den gesamten Zeitraum beobachtbar. In den anderen Ländern setzt der Rückgang erst zum Ende des 19. Jahrhunderts ein, da die Industrialisierung und die damit einhergehende Verstädterung zunächst eine Verschlechterung der Überlebenschancen von Säuglingen mit sich brachten. Im 20. Jahrhundert kommt es zu einem starken Rückgang und der Nivellierung auf sehr niedrigem Niveau. Die Abbildung 1 zeigt auch den Anstieg der Säuglingssterblichkeit in den beiden Weltkriegen und der Nachkriegszeit.

Die Differenz der Säuglingssterblichkeit zwischen Preußen und dem Süden Deutschlands weist auch auf das beträchtliche Nord-Süd-Gefälle hin, das im 19. Jahrhundert in Deutschland bestand. Generell wies der Ostseeraum eine vergleichsweise geringe Säuglingssterblichkeit auf. Dies betrifft nicht nur Länder wie Schweden oder Dänemark, sondern auch die deutschen Ostseeanrainergebiete Schleswig, Holstein, Mecklenburg und Pommern. Die Überlebensverhältnisse von Kleinkindern im 19. Jahrhundert waren aber nicht nur im Norden besser als im Süden, sondern auch im Westen tendenziell besser als im Osten. So war die Säuglingssterblichkeit im Nordwesten am geringsten und im Südosten am höchsten. Als wichtige Ursache wird die regional sehr unterschiedlich verteilte Häufigkeit und Dauer des Stillens angenommen, sowohl zwischen Nord- und Süddeutschland als auch zwischen Stadt und Land (Gehrmann 2000, 2011; Imhof 1981; Kintner 1985; Knodel 1968, 1988; Prinzing 1900). Erste regionale Untersuchungen des Kaiserlichen Statistischen Amts zur Säuglingssterblichkeit (Abb. 2) in Deutschland im Zeitraum 1875 bis 1877 zeigen für Bayern und Schwaben eine Säuglingssterblichkeit von über $30 \%$, während sie in Ostfriesland und Schleswig-Holstein unter $15 \%$ lag.

Ein in der Literatur oft erwähnter Faktor in Bezug auf regionale Säuglingssterblichkeitsunterschiede ist die Konfessionszugehörigkeit beziehungsweise mit ihr zusammenhängende Mentalitätsunterschiede. So war der Norden Deutschlands überwiegend evangelisch, der Süden mehrheitlich katholisch. Interessant ist, dass die Säuglingssterblichkeit im überwiegend evangelischen Franken deutlich geringer war als im benachbarten katholischen Bayern (/mhof 1981; Prinzing 1900). Auch saisonale klimatische Ereignisse fielen regional unterschiedlich aus und hatten einen 
Abb. 1: Säuglingssterblichkeit in ausgewählten europäischen Ländern, 18002010 (gleitender 5-Jahres-Durchschnitt)

Säuglingssterbefälle je 1.000 Lebendgeborene

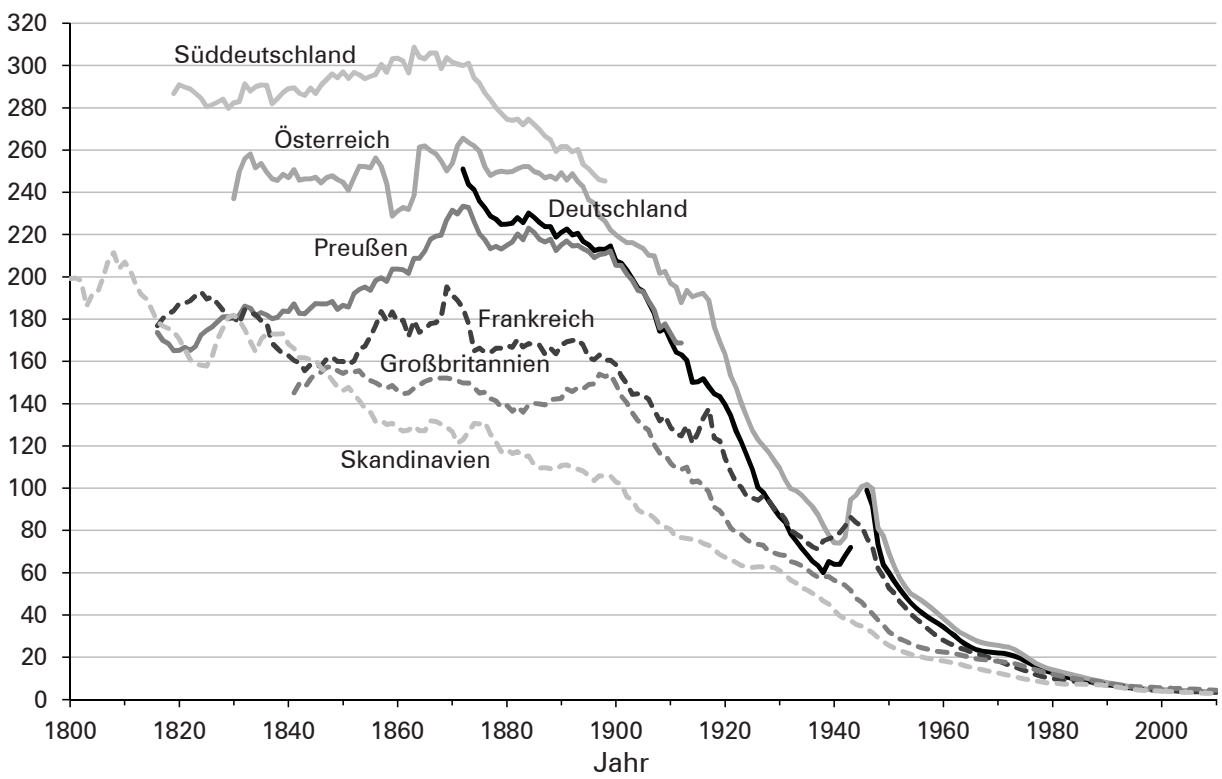

Anmerkungen: Skandinavien besteht aus den Ländern Schweden (ab 1800), Dänemark (ab 1835) und Norwegen (ab 1846); Großbritannien besteht aus England und Wales (ab 1841) und Schottland (ab 1855); Süddeutschland besteht aus Bayern (ab 1819), Sachsen (ab 1831), Baden (ab 1852), Württemberg (ab 1859 sowie 1819-1822 und 1847-1856) und dem Großherzogtum Hessen (ab 1863); Preußen (ab 1816) basiert auf dem Gebietsstand von 1866.

Quelle: Human Mortality Database (für Österreich ab 1947, Frankreich, Skandinavien und Großbritannien), Statistisches Bundesamt (für Deutschland), Gehrmann 2002 und 2011 (für Preußen), Gehrmann 2011 (für Süddeutschland) und Mitchel/ 2007 (für Österreich bis 1946); eigene Berechnung nach der Kalenderjahrmethode

Einfluss auf die Säuglingssterblichkeit speziell von nicht-gestillten Kindern (Prinzing 1899; Stöckel 1986).

Des Weiteren war die Säuglingssterblichkeit in vielen Teilen Deutschlands in den Städten höher als auf dem Lande (Spree 1988; Gehrmann 2002; Prinzing 1900). Während die Säuglingssterblichkeit auf dem Land wesentlich mit den dort herrschenden Lebens- und Arbeitsverhältnissen zusammenhing, ist das höhere Niveau in den Städten mit dem einsetzenden Urbanisierungs- und Industrialisierungsprozess zu begründen. Diese Unterschiede nahmen jedoch zum Ende des 19. Jahrhunderts und mit dem Beginn des 20. Jahrhunderts zu Gunsten der Städte ab, was auf die zuerst in den Städten erfolgten Verbesserungen der Ernährungs-, Hygiene- und Wohnbedingungen zurückzuführen ist (Gehrmann 2011; Imhof 1981; Prinzing 1899, 1900; Stöckel 1986). 
Abb. 2: Regionale Säuglingssterblichkeit in Deutschland 1875-1877 mit ausgewählten Orten: Hesel, Leezen, Zachow, Altdorf, Philippsburg, Schwalm und Gabelbach 1780-1899 (Mikrostudie)

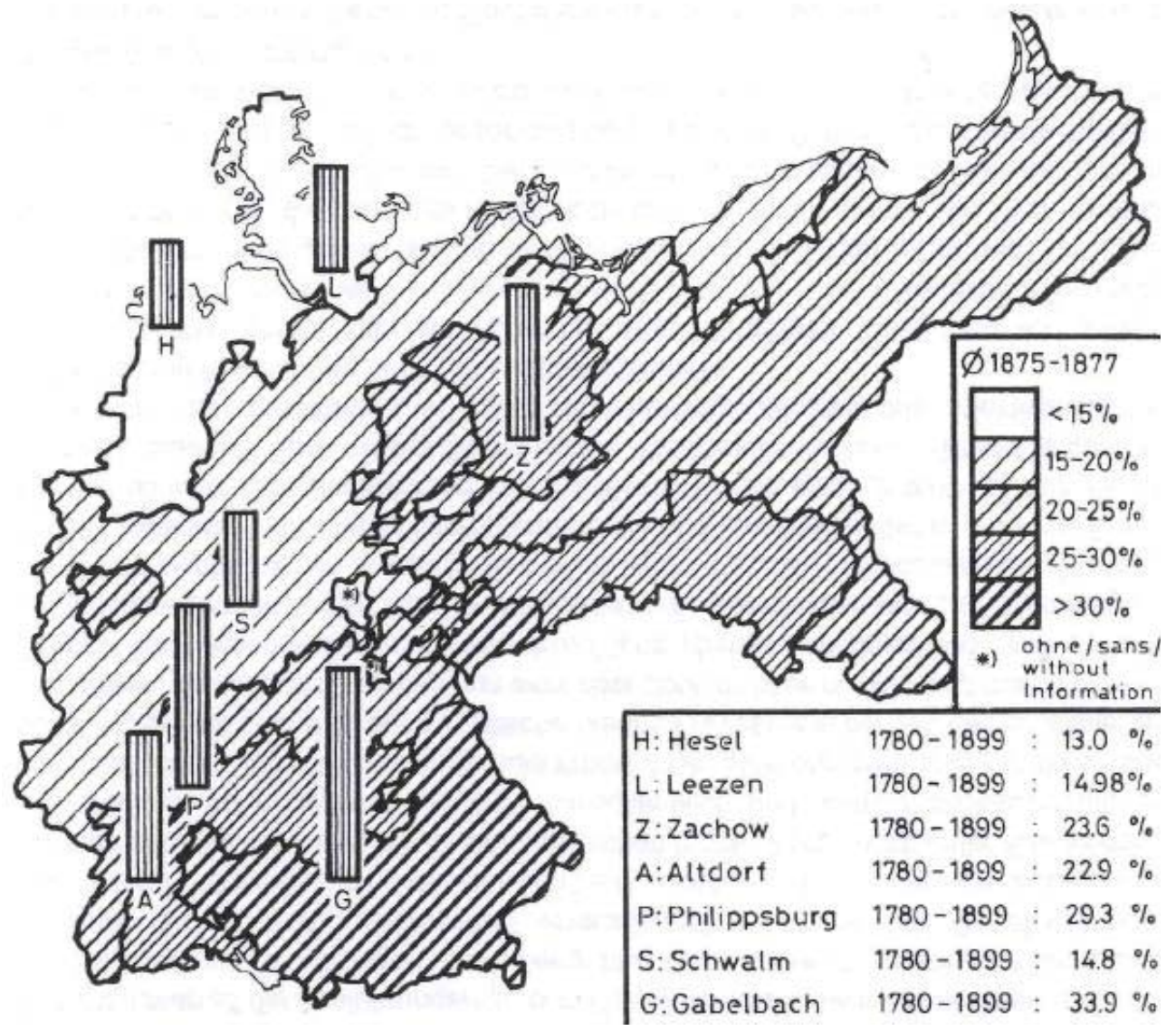

Quelle: Amtliche Regionaldaten: Würzburg (1887), Mikrostudie: Imhof (1981); Grafik: Imhof (1981)

Hinsichtlich der Analyse der Säuglingssterblichkeit in Rostock und Mecklenburg machte sich der Rostocker Pädiater Hermann Brüning verdient. Für die Hansestadt Rostock stellte er im Zeitraum 1901-1905 eine Säuglingssterblichkeit von 15,1 \% für die ehelichen bzw. von 24,2 \% für die unehelichen Lebendgeburten fest (Brüning 1908: 375). Auch fand er einen Zusammenhang zwischen sozialer Schicht und Säuglingssterblichkeit, mit einer erhöhten Sterblichkeit in niedrigen sozialen Schichten.

Unsere Studie untersucht, inwieweit zu Beginn des 19. Jahrhunderts soziale Unterschiede in der Säuglingssterblichkeit in der Rostocker Kirchgemeinde St. Jakobi bestanden. Die systematische Erschließung von historisch-demografischen Quellen über die Kirchenbuchaufzeichnungen der Geburts- und Sterberegister des 
19. Jahrhunderts ermöglicht es, Analysen vorzunehmen, wie sie mangels digitalisierter Quellen ${ }^{3}$ bislang nicht möglich waren. ${ }^{4}$

Ein besonderer Schwerpunkt liegt dabei auf dem Einfluss der Legitimität der Geburt und des Berufes des Vaters. Es wird von den Hypothesen ausgegangen, dass die Säuglingssterblichkeit ehelich geborener Kinder im Vergleich zu unehelich geborenen verringert ist und dass Säuglinge, deren Väter eine niedrige berufliche Stellung innehaben im Vergleich zu Neugeborenen mit beruflich besser gestellten Vätern ein erhöhtes Sterberisiko aufweisen.

Die Einflussfaktoren der Sterblichkeit wandeln sich über das erste Lebensjahr (Brüning 1928; Brüning/Mahlo 1929; Imhof 1981). Während die Sterblichkeit in den ersten dreißig Tagen noch stark durch die Entwicklung des Fötus während der Schwangerschaft der Mutter wie auch durch Probleme während der Geburt bestimmt ist, treten in den folgenden elf Monaten immer mehr Umwelteinflüsse in den Vordergrund. Alle Analysen werden daher separat für die ersten dreißig Lebenstage und die darauffolgenden elf Monate durchgeführt.

\section{Historische Befunde zum Einfluss sozioökonomischer Unterschiede auf die Säuglingssterblichkeit}

Die Einflussfaktoren auf die Säuglingssterblichkeit im 19. Jahrhundert sind vielfältig und schwer voneinander zu trennen, da sie sich zum Teil gegenseitig bedingen. Gerade die regionalen Unterschiede der Säuglingssterblichkeit waren im 18. und vor allem im 19. Jahrhundert in Deutschland wesentlich größer als heutzutage; eine umfassende Erklärung dieser Unterschiede ist jedoch in Anbetracht der schwierigen Quellenlage und der Komplexität der Ursachen, die weit über das Fachgebiet der Historischen Demografie hinausgehen, bislang nicht zufriedenstellend erfolgt (Ehmer 2004: 92-94).

Imhof (1981) führt in einem Artikel, der sich auf jahrelange eigene Forschung sowie Arbeiten von Bluhm (1912), Krieg (1890), Prinzing (1899, 1900, 1931), Würzburg $(1887,1888)$ u.a. sowie amtliche Daten stützt, die wichtigsten Einflussfaktoren an. Diese sind Saisonalität, Regionalität, Häufigkeit und Dauer des Stillens, Krisenzeiten, Legitimität der Geburt, Geburtsgewicht, Geburtsrang, Familiengröße, Geburtenabstände, Geschlecht, Alter der Mutter, Schichtenzugehörigkeit, Arbeits-, Wohnungs-, Ernährungs- und Hygienebedingungen sowie Konfession und Mentalität der Eltern.

3 Das Land Mecklenburg-Vorpommern förderte für zwei Jahre den Rostocker Forschungsverbund Historische Demografie - eine Kooperation der Universität Rostock mit dem Max-PlanckInstitut für demografische Forschung. Dabei wurde die Digitalisierung von handschriftlichen Urquellen der Volkszählungen Mecklenburg-Schwerins aus den Jahren 1867 und 1900 sowie ausgewählten demografischen Bewegungsmengen (Geburten, Sterbefälle, Eheschließungen) von Rostocker Kirchenbüchern in dem Zeitraum 1800 bis 1900 durchgeführt.

4 Mühlichen (2011), Masterarbeit, Universität Rostock. 
Im Folgenden sollen speziell die sozioökonomischen Faktoren näher beleuchtet werden, wenngleich für Säuglingssterblichkeitsunterschiede verantwortliche Verhaltensmuster nicht allein sozial erklärt werden können (Spree 1980). Ein umfassenderer Überblick über den Forschungsstand zu anderen Einflussfaktoren in Deutschland im 19. Jahrhundert findet sich u.a. bei Imhof (1981) und Gehrmann (2011). Zu den sozioökonomischen Faktoren zählen wir die Legitimität der Geburt, Schichtzugehörigkeit und Arbeitsbedingungen.

Die Legitimität der Geburt unterscheidet, ob ein Säugling innerhalb einer Ehe oder außerehelich geboren wurde. Dieser Faktor ist von großer Bedeutung, da bei unehelichen Kindern in der zeitgenössischen bevölkerungsstatistischen und medizinischen Literatur (z.B. Prinzing 1900; 1911; Saul 1909) eine wesentlich höhere Sterblichkeit im Säuglingsalter nachgewiesen wird als bei ehelichen Kindern. Zwar würde ein Teil der unverheirateten Frauen die Geburt nachträglich durch eine Hochzeit legitimieren, jedoch treffe dies nicht auf die Mehrheit zu (Prinzing 1902: 44). Ursächlich für die höhere Sterblichkeit illegitimer Säuglinge war insbesondere der Mangel an Fürsorge durch die vergleichsweise ungünstige finanzielle Situation, welche die Mütter zwang, arbeiten zu gehen und das Kind an Vertraute oder eine Institution zu geben (Vögele 1994: 411; Preston/Haines 1991: 30). Allerdings war der Einfluss der Legitimität auf die Säuglingssterblichkeit im Vergleich zum Einfluss der Ernährungs-, Arbeits- und Hygienebedingungen eher gering (Kintner 1994; Spree 1998).

Zum Einfluss der Arbeitsbedingungen von Vätern und Müttern - in Form von beruflicher Schicht, Einkommen und Arbeitsbelastung - auf die Sterblichkeit ihrer Kinder im ersten Lebensjahr gibt es national wie international unterschiedliche Ergebnisse. Bengtsson und Lundh (1994) können für Skandinavien keine Wirkung des Reallohns auf die Säuglingssterblichkeit nachweisen, während Sundin (1995) für Schweden zumindest in Zeiten eines besonders raschen Rückgangs der Säuglingssterblichkeit speziell die Oberschicht als Profiteure sieht. Woods et al. $(1988,1989)$ und Haines (1995) können für England und Wales hingegen einen Einfluss der sozialen Schicht und des Einkommens feststellen, zumindest auf die Höhe der Säuglingssterblichkeit, aber weniger auf den Zeitpunkt und das Ausmaß des Säuglingssterblichkeitsrückgangs im späten 19. und frühen 20. Jahrhundert. Im Vergleich zu Frankreich erkennt Woods (1994) jedoch speziell in den stärker ausgeprägten Stillgewohnheiten, niedrigeren Fertilitäts- und Illegitimitätsraten sowie dem weitgehenden Ausbleiben von Krisenzeiten die Gründe für die geringere Säuglingssterblichkeit Englands. Auch deutsche Zeitgenossen wie Prinzing (1899) und Hanssen (1912) sehen in den Stillpraktiken größere Erklärungskraft als in sozioökonomischen Faktoren, wenngleich Prinzing (1899: 588) zumindest für die West-Ost-Unterschiede Preußens die besseren ökonomischen und strukturellen Bedingungen in den westlichen Provinzen als Ursache sieht. Hanssen (1912: 8) schreibt, dass ein Einfluss der sozialen Schicht in Schleswig-Holstein nur für ungestillte Säuglinge messbar sei. Bei Betrachtung der benachbarten Region Mecklenburg-Schwerin stellt Saul (1909: 38) jedoch fest, dass die Säuglingssterblichkeit für Kinder von ökonomisch schlechter gestellten Arbeitern wie Tagelöhnern, Fabrikarbeitern oder Knechten höher war im Vergleich zu den finanziell besser gestellten Erwerbstätigen. Von Bedeutung ist auch die Arbeitsbelastung der Frauen. Speziell die Fabrikarbeit von Frauen hatte 
einen negativen Einfluss auf die Überlebenschancen der Säuglinge, fand jedoch im Mecklenburg des 19. Jahrhunderts im Vergleich zu den industrialisierten Gebieten Deutschlands praktisch nicht statt. Aber auch die Einbindung der Mütter in landwirtschaftliche Arbeit wirkte sich negativ aus (Gehrmann 2011; Heller/Imhof 1983; Prinzing 1899). Sozioökonomische Faktoren üben also insofern einen Einfluss auf die Säuglingssterblichkeit aus, dass sie das Stillverhalten und die Qualität der Ernährung sowie Pflege und Fürsorge beeinflussen (Imhof 1981: 359; Kloke 1997: 76).

Ein weiterer Einflussfaktor ist die Mentalität in Form von "regional, konfessionell, sozial unterschiedliche[n] Einstellungen von Bevölkerungen zur Fruchtbarkeit, zur Geschlechtlichkeit, zu Gesundheit und Krankheit, zum Sterben und Tod" IImhof 1981: 366). Kriege und Krisenzeiten z.B. haben die Wertschätzung des Lebens vermutlich eher negativ beeinflusst und so war die Säuglingssterblichkeit gerade in Gebieten mit einer hohen Krisen- und Kriegshäufigkeit verhältnismäßig hoch (Imhof 1981: 367). Imhof (1981: 375) macht zwei zueinander konträre Mentalitäten aus: Das "System der Bewahrung menschlichen Lebens" und das "System der Verschwendung". Ersteres ist durch eine allgemeine Wertschätzung des Lebens geprägt, die beispielsweise durch das lange Stillen des Säuglings zum Ausdruck kommt, während letzteres - durch Traumata und Krisenzeiten geprägt - dem Leben eine gewisse Gleichgültigkeit entgegenbringt und für den Süden Deutschlands kennzeichnender war als für den Norden. „Diese Gleichgültigkeit war eine direkte und unausweichliche Konsequenz der Demographie der Epoche." (Ariés 1975: 99)

Im Hinblick auf die Konfession konnten in Süddeutschland für die protestantischen Regionen oft deutlich niedrigere Säuglingssterbeziffern ausgemacht werden als in katholischen Gebieten. Ein gutes Beispiel ist in diesem Zusammenhang das Königreich Bayern (Prinzing 1900: 620). Als Erklärungsversuch führt Imhof (1981: 378-379) die unter Katholiken zur damaligen Zeit verbreitete Vorstellung vom so genannten „Himmeln“ an: Um in armen, kinderreichen Familien das Überleben wenigstens der älteren Geschwister zu sichern, wurde mitunter eine Vernachlässigung des jüngsten Kindes und somit auch dessen Tod in Kauf genommen, auch um ihm das Leid unter den ärmlichen Verhältnissen zu ersparen und ihm als noch sündlosem Nachkommen zudem einen Platz im Himmel zu ermöglichen, von dem aus es wiederum seiner Familie einen Platz dort sichern könne. Andererseits konnten in religiös gemischten Gebieten keine signifikanten Unterschiede in der Säuglingssterblichkeit zwischen den beiden christlichen Konfessionen festgestellt werden (Kloke 1997: 266). Die Unterschiede in Bayern sind auch eher im Zusammenhang mit dem damaligen deutschen Nord-Süd-Gefälle zu sehen. Zudem wies gerade das evangelische Laichingen in Württemberg mit einem Niveau von zeitweise 40 bis $50 \%$ besonders hohe Werte für die Säuglingssterblichkeit auf (Medick 1997: 359-365). Auch in den Niederlanden konnte ein solches Nord-Süd-Gefälle im späteren 19. Jahrhundert gefunden werden, zu Ungunsten der mehrheitlich katholischen Gebiete im Süden, welches mit einer deutlich geringeren Stillneigung und einer größeren Skepsis gegenüber neuen Ideen zur Krankheitsbekämpfung und Hygieneverbesserung im Vergleich zum evangelisch dominierten Norden erklärt werden kann (Wolleswinkelvan den Bosch et al. 2000). 
Ein weitaus größerer Einfluss wird in der Literatur daher den regional nachweislich unterschiedlichen Ernährungs- und Stillgewohnheiten zugeschrieben. So stellen beispielsweise Imhof (1981: 347-349, 353-354), Kloke (1997: 54, 157, 266) und Gehrmann (2002: 546) heraus, dass die Überlebenschancen von Säuglingen in Regionen, in denen häufig und lange gestillt wurde, deutlich besser waren als in Gebieten, in denen das nicht der Fall war. „In den norddeutschen Dörfern mit einer geringen Säuglingssterblichkeit scheinen nahezu alle Mütter ihre Säuglinge gestillt zu haben, und dies auch relativ lang, zumindest für das ganze erste Lebensjahr. In süddeutschen Gemeinden mit einer hohen Säuglingssterblichkeit wurde dagegen schon von den Zeitgenossen darüber geklagt, dass viele Mütter ihre Kinder nicht oder nur ganz kurz stillen würden." (Ehmer 2004: 94) Gerade im Sommer war die Säuglingssterblichkeit aufgrund von Magen- und Darmerkrankungen, die auf schlechte, durch die Wärme schneller verderbliche Ersatznahrung zurückzuführen waren, stark erhöht (Kloke 1997). Lee und Marschalck (2002) konnten für die Stadt Bremen im Zeitraum 1861 bis 1863 und 1870 bis 1872 in den Lebensmonaten 3, 6, 9 und 12 einen Anstieg der Mortalität der Neugeborenen feststellen, den sie damit begründen, dass genau zu diesen Zeiten häufig das Stillen beendet wurde. Laut Imhof (1981: 373) war unter den Müttern zu der Zeit durchaus nicht unbekannt, dass die Muttermilch das gesündeste Nahrungsmittel für den Säugling ist. Dass in vielen Regionen dennoch wenig gestillt wurde, könne mit mangelnder Fürsorgezeit für das Kind zusammenhängen, speziell wenn die Mutter ledig und berufstätig war oder in die Arbeit des Mannes, z.B. in einem landwirtschaftlichen Betrieb, eingebunden war. Auch das o.g. "System der Verschwendung" könne nach Imhof ein Erklärungsansatz sein.

Zum Ende des 19. Jahrhunderts setzte schließlich eine Verbesserung der Ernährungs-, aber auch der Hygiene- und Wohnbedingungen ein, die zu einem Anstieg der Überlebenschancen der Säuglinge führte, insbesondere aufgrund des damit einhergehenden Rückgangs der Magen- und Darmkrankheiten (Gehrmann 2011; Imhof 1981).

\section{Daten und Methode ${ }^{5}$}

Die vorliegende Studie basiert auf den neu digitalisierten Beerdigungs- und Taufregistern der Kirchenbücher von St. Jakobi, welche die notwendigen Informationen zu den Geburten und Sterbefällen liefern. Die Jakobi-Kirche war die mit Abstand größte der damaligen vier Rostocker Kirchspiele, zu denen auch St. Marien, St. Nikolai und St. Petri gehörten (Szołtysek et al. 2010: 10). ${ }^{6} \mathrm{Im}$ Jahr 1905 waren sogar mehr

5 Ausführliche Beschreibungen zu den Quellen und Daten siehe Mühlichen/Scholz (2015).

6 Wie der Großteil Norddeutschlands war auch die Stadt Rostock im 19. Jahrhundert überwiegend evangelisch. Eine katholische Kirche gab es erst ab 1909 wieder. St. Jakobi, Rostocks damals größte Kirche, existiert inzwischen nicht mehr. Große Teile der Kirche wurden im Jahr 1942 durch einen britischen Bombenangriff zerstört. Die noch vorhandene Bausubstanz wurde 1960 abgerissen (Köppe 2010; Kuzia 2004). 
als die Hälfte von Rostocks Einwohnern Mitglieder der Jakobikirche, woraufhin noch im gleichen Jahr aufgrund der ungenügenden Betreuungsmöglichkeit die Teilung der Gemeinde und 1908 schließlich die Fertigstellung der Heilig-Geist-Kirche in der Kröpeliner-Tor-Vorstadt vollzogen wurden (Schulz 2008: 24). Grundlage der Studie ist eine Vollerhebung aller Geburten und Sterbefälle in Rostock-St. Jakobi.

Da sowohl die Tauf- als auch die Beerdigungsregister die vollständigen Namen enthalten und die Einzelpersonen somit identifizierbar sind, ist eine Zusammenführung von Tauf- und Beerdigungsdatensatz zu einem Gesamtdatensatz möglich. Diese Zusammenführung ist für den Zeitraum 1815 bis 1829 erfolgt und dient den folgenden Analysen als Datengrundlage. Das Beispiel in Abbildung 3 zeigt links in der zweiten Zeile den Eintrag im Taufregister zu Emma Krüger, die am 28. Januar 1815 geboren und am 5. Februar des gleichen Jahres getauft wurde. Rechts, im Beerdigungsregister ist in der zweiten Zeile der Eintrag zu lesen, dass sie am 31. Dezember 1815 gestorben ist und am 5. Januar 1816 beerdigt wurde. Weitere Informationen aus dem Taufregister sind der Beruf und Name des Vaters und der Name der Mutter sowie die bei der Taufe anwesenden Gevattern. Das Beerdigungsregister enthält neben dem Namen und Beruf des Vaters auch das Alter bei Todeseintritt und die Todesursache. Auch der Geburtsort wird zum Teil angegeben, sofern es sich hierbei nicht um Rostock handelt. Informationen über eine Erwerbstätigkeit der Mutter sind nicht in den Registern enthalten.

Als Risikopopulation für die Sterblichkeitsanalysen gelten alle Geburten, die in Rostock zu St. Jakobi in den entsprechenden Untersuchungszeiträumen als Lebendgeburt registriert wurden. Zugewanderte Säuglinge, die nicht im Taufregister von St. Jakobi erfasst sind, aber im Beerdigungsregister, sind aus den Analysen ausgeschlossen (20 Fälle; $6 \%$ ). Ob und wie viele Neugeborene die Stadt verlassen oder das Kirchspiel gewechselt haben und im ersten Lebensjahr verstorben sind, kann nicht überprüft werden. Eine Untererfassung der Säuglingssterblichkeit ist hierdurch möglich. Da es weiterhin im 19. Jahrhundert noch keine einheitlichen

Abb. 3: Ausschnitte aus den Tauf- und Beerdigungsregistern von St. Jakobi, Rostock

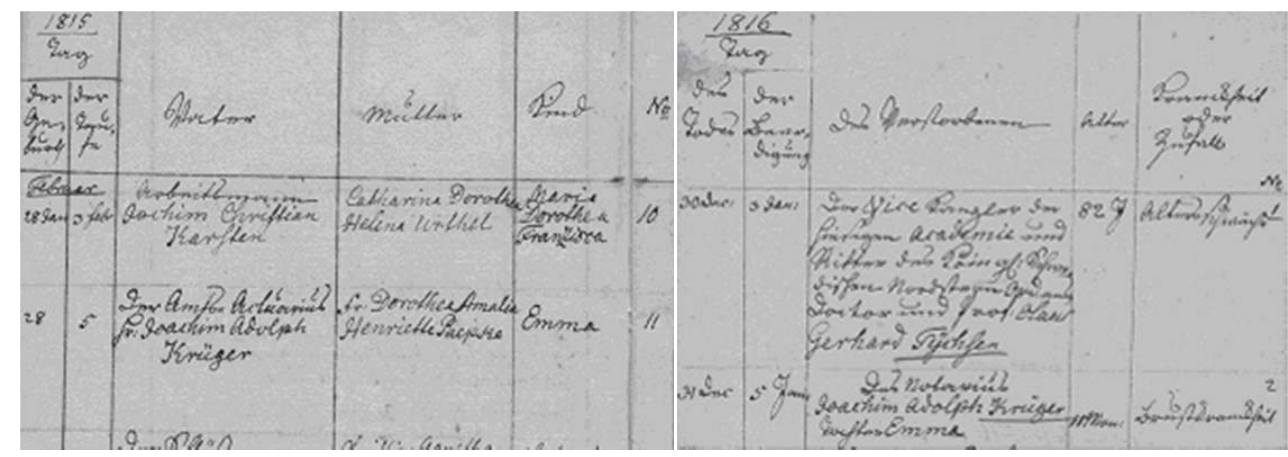

Quelle: Taufregister von St. Jakobi, Rostock, 1815 und Beerdigungsregister von St. Jakobi, Rostock, 1816 
Regeln zur Abgrenzung von Lebend- und Totgeburten gab, sind auch hierdurch Verzerrungen nicht auszuschließen.

Die Sterbeintensität im ersten Lebensjahr wird mittels Methoden der Ereignisdatenanalyse analysiert (Allison 1984; Diekmann/Mitter 1984; Blossfeldet al. 1986), wobei das Alter in Tagen die Prozesszeit misst. Diese beginnt zum Zeitpunkt der Geburt im Alter von 0 Tagen und endet mit dem Eintritt eines Sterbefalls im ersten Lebensjahr oder - im Fall einer Zensierung - mit dem ersten Geburtstag und dem dadurch bedingten Ausscheiden aus der Risikopopulation. Das Alter der Neugeborenen, die nicht innerhalb des ersten Lebensjahres sterben, wird auf den Zensierungszeitpunkt von 365 Tagen gesetzt.

Um den Einfluss der Charakteristiken der Neugeborenen auf ihr Sterberisiko zu ermitteln, werden Cox-Modelle mit proportionalem Hazard der Form $h(t \mid X)=h_{o}(t) \cdot e^{\beta x}$ gerechnet. Dabei kennzeichnet $h_{0}(t)$ den semiparametrischen Baseline Hazard, $\beta$ die zu schätzenden Parameter und $x$ die Kovariaten. Die Potenz $e^{\beta}$ ist der Hazard Ratio, welcher sich aus dem Verhältnis zweier zu vergleichender Hazardraten berechnet und zur Interpretation als relatives Risiko betrachtet wird.

Als Kovariaten gehen Geschlecht, Jahreszeit der Geburt, Legitimität der Geburt und Beruf des Vaters ein. Nach abgeschlossener Datenaufbereitung enthält der Datensatz 2.768 Lebendgeburten im Zeitraum 1815 bis 1829, von denen 331 im ersten Lebensjahr verstorben sind, was einem Anteil von 12,0 \% entspricht. Des Weiteren gibt es 124 Totgeburten. Der Anteil der Totgeburten an allen Geburten zwischen 1815 und 1829 beträgt 4,3\%. Totgeborene sind aber aus allen Analysen und Tabellen ausgeschlossen.

Das Geschlecht ist männlich und weiblich kategorisiert, die Saisonalität bei Geburt hat die Ausprägungen Frühling, Sommer, Herbst und Winter. Für die Einteilung der Jahreszeiten wurde auf die meteorologische Kategorisierung zurückgegriffen mit Frühling von März bis Mai, Sommer von Juni bis August, Herbst von September bis November und Winter von Dezember bis Februar.

Die Legitimität der Geburt ist unterteilt in ehelich und unehelich. Die Legitimität taucht nicht als eigene Spalte in den Registern auf. Ob eine Geburt unehelich ist, kann an Vermerken wie „unehelich“ oder „angeblicher Vater ist [...]” erkannt werden.

Zur Operationalisierung der Berufe haben wir basierend auf der Klassifizierung des North Atlantic Population Project (NAPP 2013) sowie den Studien zur historischen Erwerbs- und Sozialstruktur im deutschen Ostseeraum (Brandenburg et al. 1991; Brandenburg/Kroll 1998; Lorenzen-Schmidt 1996; Manke 2000) eine neue Klassifizierung entworfen, die es sowohl ermöglicht, die Häufigkeiten verschiedenartiger Tätigkeiten auszuzählen als auch diese nach Berufsgruppen oder nach sozialer Schicht zusammenzufassen (Mühlichen/Scholz 2015). Nach sozialer Schicht aggregiert, ergeben sich folgende Obergruppen für den Beruf des Vaters: 1) Tätigkeiten mit gehobener Stellung, 2) Tätigkeiten mittlerer Stellung, 3) Tätigkeiten niedriger Stellung und 4) sonstige Tätigkeiten oder unbekannt. Die erste Kategorie umfasst Kaufmänner, Händler, Juristen, gehobene Beamte, Adlige, Mediziner, Lehrer, Professor, Pfarrer, Pensionäre, Bierbrauer und Handwerksmeister. In der zweiten Ausprägung sind Handwerker, Rathaus- und Gerichtsdiener, Gastwirte und Schiffer vertreten. Die dritte Gruppe bilden Handwerksgesellen, Lohndiener, Träger, 
Ackersmänner, Gärtner, Fischer, Musikanten, Seefahrer, Matrosen, Steuermänner, einfache Soldaten und Erwerbslose. In der vierten Ausprägung sind vor allem Arbeitsmänner enthalten, die eine im Hinblick auf die Art der Tätigkeit und in finanzieller Hinsicht sehr heterogene Gruppe darstellen, und Väter, über die nichts bekannt ist. Auch vor der Geburt verstorbene Väter sowie "uneheliche" oder "angebliche" Väter sind in dieser Kategorie enthalten. Diese vierte Gruppe stimmt daher auch fast zur Hälfte mit der Kategorie "unehelich" der Legitimitätsvariable überein. Eine Ausnahme stellen uneheliche Väter dar, die die Mutter des Kindes innerhalb des Beobachtungszeitraums geheiratet haben, was an weiteren gemeinsamen Geburtseinträgen im Datensatz erkennbar ist: Sie sind in der jeweils ihrem Beruf zuzuordnenden Schicht anzutreffen (insgesamt 12 Fälle).

Tabelle 1 enthält eine Übersicht der Kovariaten nach ihren Ausprägungen, der Anzahl der Sterbefälle, Zensierungen, Risikozeiten und Sterberaten. Es wird nach Sterblichkeit in den ersten dreißig Lebenstagen sowie zwischen dem zweiten und

Tab. 1: Sterbefälle, Zensierungen, Personenjahre und Sterberate je nach Ausprägung der unabhängigen Variablen im Ereignisdatensatz zur Säuglingssterblichkeit in Rostock im Zeitraum 1815-1829

\begin{tabular}{|c|c|c|c|c|c|c|}
\hline \multirow[t]{2}{*}{ Variable } & \multicolumn{3}{|c|}{ Sterbefälle } & \multirow[t]{2}{*}{ Zensierungen } & \multirow{2}{*}{$\begin{array}{l}\text { Personen- } \\
\text { jahre }\end{array}$} & \multirow[t]{2}{*}{ Sterberate } \\
\hline & Gesamt & 1. Monat & 2.-12. Monat & & & \\
\hline \multicolumn{7}{|l|}{ Geschlecht } \\
\hline Männlich & 175 & 67 & 108 & 1292 & 1339 & 0,1307 \\
\hline Weiblich & 156 & 53 & 103 & 1145 & 1191 & 0,1310 \\
\hline Gesamt & 331 & 120 & 211 & 2437 & 2530 & 0,1308 \\
\hline \multicolumn{7}{|l|}{ Saisonalität bei Geburt } \\
\hline Frühling & 84 & 23 & 61 & 602 & 626 & 0,1341 \\
\hline Sommer & 80 & 35 & 45 & 591 & 612 & 0,1307 \\
\hline Herbst & 85 & 33 & 52 & 599 & 621 & 0,1368 \\
\hline Winter & 82 & 29 & 53 & 645 & 670 & 0,1224 \\
\hline Gesamt & 331 & 120 & 211 & 2437 & 2530 & 0,1308 \\
\hline \multicolumn{7}{|l|}{ Beruf des Vaters } \\
\hline Gehobene Tätigkeit & 51 & 16 & 35 & 455 & 469 & 0,1087 \\
\hline Mittlere Tätigkeit & 113 & 43 & 70 & 738 & 768 & 0,1472 \\
\hline Einfache Tätigkeit & 81 & 32 & 49 & 452 & 474 & 0,1707 \\
\hline Sonstige, unbekannt & 86 & 29 & 57 & 792 & 819 & 0,1051 \\
\hline Gesamt & 331 & 120 & 211 & 2437 & 2530 & 0,1308 \\
\hline \multicolumn{7}{|l|}{ Legitimität der Geburt } \\
\hline Ehelich & 290 & 107 & 183 & 2063 & 2144 & 0,1353 \\
\hline Unehelich & 41 & 13 & 28 & 374 & 386 & 0,1063 \\
\hline Gesamt & 331 & 120 & 211 & 2437 & 2530 & 0,1308 \\
\hline
\end{tabular}

Quelle: Tauf- und Beerdigungsregister der Kirchenbücher von St. Jakobi, Rostock (eigene Berechnungen) 
zwölften Lebensmonat unterschieden. Die Sterberate berechnet sich aus der Sterbefallzahl im ersten Lebensjahr geteilt durch die Personenjahre.

Die komplette Dateneingabe, die Fehlerkorrektur und die Zusammenführung der Tauf- und Beerdigungsdaten wurden mit Microsoft Excel durchgeführt. Für die Datenaufbereitung zur Survivalanalyse wurde das statistische Programmpaket SPSS 20 verwendet. Die Ereignisdatenanalyse wurde mit STATA 11 ausgeführt.

\section{$4 \quad$ Ergebnisse}

Abbildung 4 zeigt die Entwicklung der Säuglingssterblichkeit in der Rostocker Kirchgemeinde St. Jakobi im 19. Jahrhundert. Der Untersuchungszeitraum des Ereignisdatenanalysemodells (1815 bis 1829) ist darin umrandet. ${ }^{7}$ Bis 1840 ist die Mortalität der Neugeborenen auf einem in etwa gleich bleibenden bis minimal sinkenden $\mathrm{Ni}$ veau. Sie schwankt im Untersuchungszeitraum 1815 bis 1829 zwischen 104 und 143 Sterbefällen je 1.000 Lebendgeborene und beträgt 127,7 für Jungen und 127,9 für Mädchen, für beide Geschlechter insgesamt 127,8. Nennenswerte Geschlechterunterschiede lassen sich erst ab 1830 zu Ungunsten der Jungen ausmachen. Ab 1840 steigt außerdem das Niveau der Säuglingssterblichkeit erheblich an, bis auf 219 Todesfälle je 1.000 Lebendgeborene im Jahr 1858. Die Ursachen hängen vermutlich mit der gestiegenen Bevölkerungszahl und deren Folgen auf die Wohn-, Arbeits-, Ernährungs- und Hygienesituation zusammen. Die Säuglingssterblichkeit geht in der Folge wieder leicht zurück und schwankt zwischen 1880 und 1902 stagnierend um den Wert 170.

In Abbildung 5 sind die Kaplan-Meier-Überlebensverläufe für die unabhängigen Variablen Legitimität der Geburt und Beruf des Vaters dargestellt.

Im Hinblick auf die Legitimität der Geburt weisen die unehelichen Geburten einen günstigeren Überlebensverlauf auf als die ehelichen Geburten. Das erste Lebensjahr überleben $90 \%$ der unehelichen und $88 \%$ der ehelich Geborenen. Die Ursachen hierfür werden im nächsten Kapitel diskutiert.

Für die Berufsgruppen der Väter ergeben sich deutlichere Unterschiede. So gestaltet sich der Überlebensverlauf im ersten Lebensjahr für Kinder, deren Väter einer einfachen Tätigkeit nachgehen, mit $85 \%$ deutlich schlechter im Vergleich zu den anderen Berufsgruppen. Der Anteil Überlebender liegt für Kinder von Vätern mittlerer beruflicher Schicht bei 87 \% und bei Kindern von Vätern mit gehobener Tätigkeit bei $90 \%$. Ebenfalls $90 \%$ erreicht auch die Kategorie von Säuglingen, bei denen die Tätigkeit des Vaters nicht bekannt oder nicht zuordenbar ist oder der Vater selbst unbekannt ist. Diese Gruppe stimmt in ihrer Zusammensetzung fast zur Hälfte mit der Kategorie „unehelich“ der Legitimitätsvariable überein. Wegen dieser Multikollinearität werden die beiden Variablen in getrennten Modellen untersucht.

7 Die 20 Sterbefälle Zugewanderter im Zeitraum 1815 bis 1829 sind in diese Darstellung einbezogen, um eine kontinuerliche Zeitreihe darzustellen (für die Jahre ab 1830 erfolgte noch keine entsprechende Aufbereitung, die den Ausschluss von Zuwanderung ermöglicht). In den folgenden Analysen sind diese Fälle ausgeschlossen. 
Abb. 4: Säuglingssterblichkeit* in Rostock-St. Jakobi nach Geschlecht, 18001902 (gleitender 3-Jahres-Durchschnitt)

Säuglingssterbefälle je 1.000 Lebendgeborene

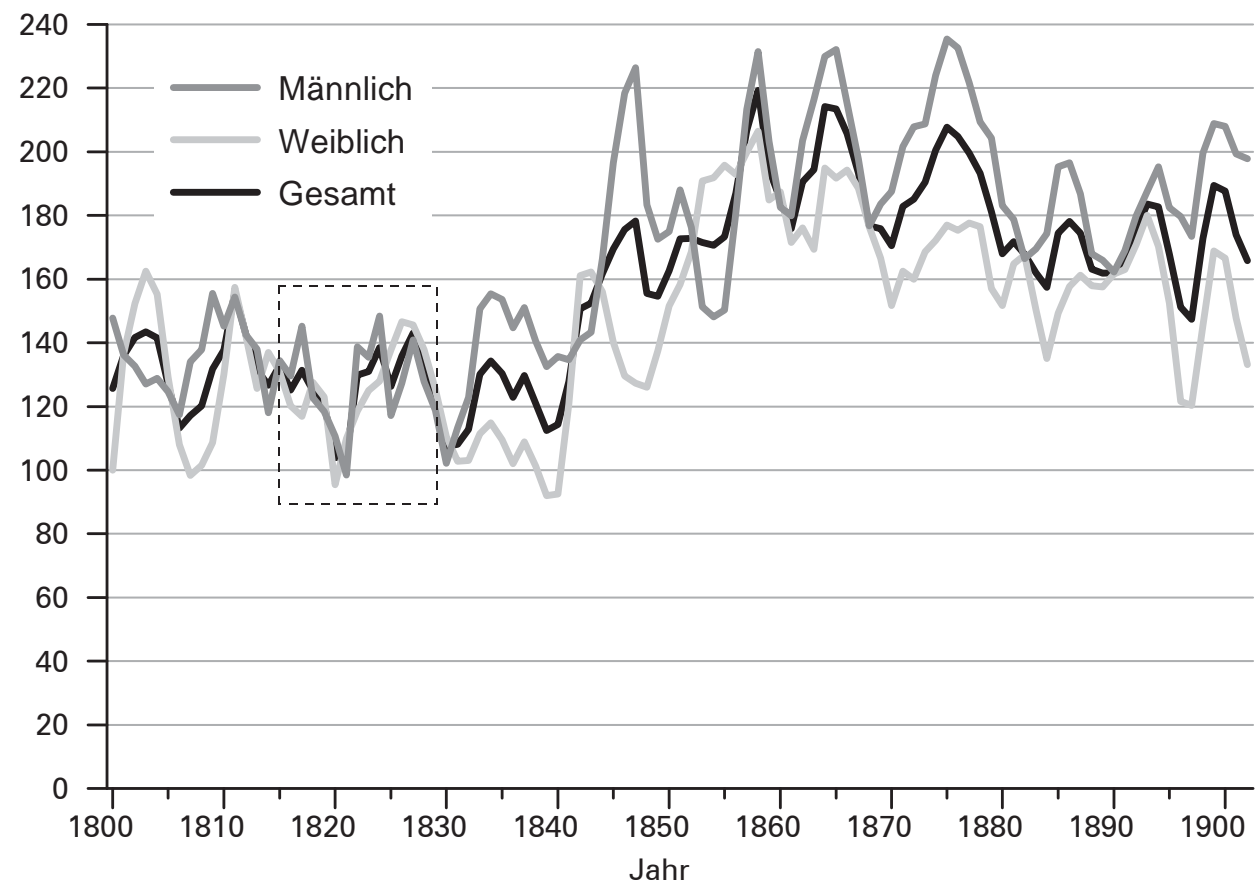

* Für den Analysezeitraum 1815 bis 1829 konnte die Säuglingssterblichkeit nach der Geburtsjahrmethode von Becker (1874) und Zeuner (1869) berechnet werden. Für die übrigen Jahre wurde die Kalenderjahrmethode von Böckh verwendet (Esenwein-Rothe 1982: 241-248). Die Sterbefälle eines Jahres $t$ wurden jedoch nicht zu $100 \%$, sondern zu $70 \%$ den Geburten des Jahres t und zu 30 \% den Geburten des Jahres t+1 zugeordnet. Diese vorgenommene Gewichtung erscheint nach entsprechender Auswertung der Jahre 1815 bis 1829 realistischer. Die zweitweise sehr großen Geschlechterunterschiede (z.B. im Jahr 1847) können zum Teil ein Resultat der ungenaueren Kalenderjahrmethode sein. Die Geburtsjahrmethode ist insbesondere bei kleinen Fallzahlen wegen ihres Kohortenansatzes genauer.

Quelle: Tauf- und Beerdigungsregister der Kirchenbücher von St. Jakobi, Rostock (eigene Berechnungen)

Niedrige Fallzahlen sind für den unregelmäßigen Verlauf der Überlebenskurven verantwortlich. Da sich die Kurven der Berufsgruppen mehrfach schneiden, liegt zudem die Vermutung nahe, dass die Proportionalitätsannahme des Cox-Proportionalen-Hazard-Modells nicht erfüllt ist. Der Loglogsurvival-Plot (Abb. 6) bestätigt dies, da die Kurven nicht parallel verlaufen. In einem ersten Schritt soll aus Gründen der Vergleichbarkeit der Einfluss der Kovariaten auf die Sterblichkeit im gesamten ersten Lebensjahr ermittelt werden (Tab. 2). 
Abb. 5: Kaplan-Meier-Überlebensverlauf für das erste Lebensjahr der zwischen 1815 und 1829 in Rostock zu St. Jakobi geborenen Säuglinge nach Legitimität der Geburt (links) und Beruf des Vaters (rechts)
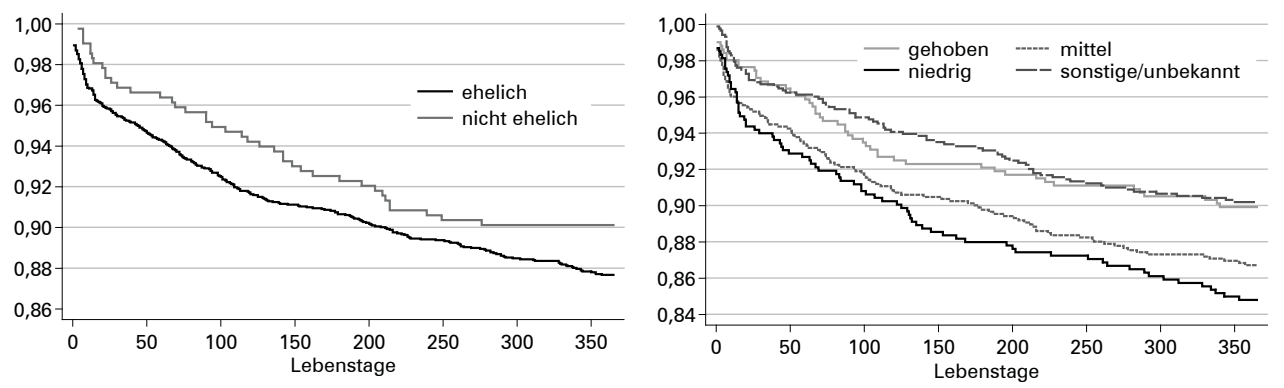

Abb. 6: Test der Proportionalitätsannahme (Loglogsurvival-Plot) für die unabhängigen Variablen Legitimität der Geburt (links) und Beruf des Vaters (rechts)
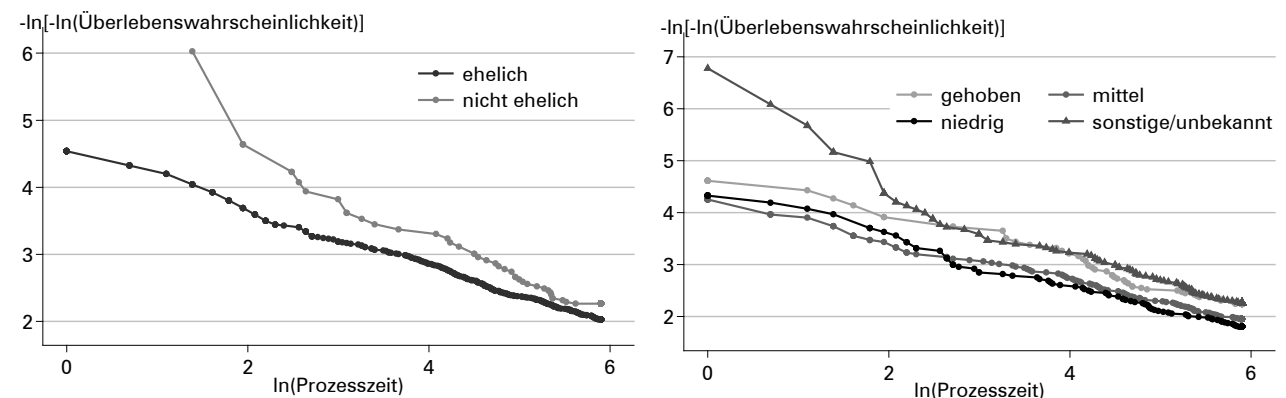

Quelle: Tauf- und Beerdigungsregister der Kirchenbücher von St. Jakobi, Rostock (eigene Berechnungen)

Beim Vergleich der vier Modelle für das erste Lebensjahr fällt auf, dass sich durch das schrittweise Hinzufügen neuer Einflussgrößen die relativen Risiken nicht ändern. Die Legitimität der Geburt und der Beruf des Vaters werden in separaten Modellen betrachtet, da die Kategorie der unehelichen Geburten zur Hälfte identisch ist mit der Kategorie ,sonstige/unbekannt' des Berufs des Vaters.

Bei den Berufsgruppen wird der Eindruck aus den Kaplan-Meier-Überlebensverläufen (Abb. 6) bestätigt. Im Vergleich zur Referenzgruppe, den Kindern von Vätern mit gehobener Tätigkeit, ist das relative Risiko, das erste Lebensjahr nicht zu überleben, für die Kinder von Vätern mittlerer Berufsschicht um $34 \%$, für die Kinder von Vätern niedriger beruflicher Tätigkeit um $55 \%$ erhöht. Diese Unterschiede sind signifikant. Die Restkategorie unterscheidet sich nicht signifikant von der Referenzgruppe. Über diese Obergruppen hinaus ist das Risiko bei den Berufsgruppen der Rathaus- und Gerichtsdiener, Musikanten, Fischer, Gastwirte, Matrosen und Seefahrern sowie Ackersmännern am höchsten, während es bei höheren Beamten und Akademikern, Kaufleuten, Arbeitsmännern und Trägern am geringsten ist. Die groBe Gruppe der Handwerker, sowohl der Meister als auch Gesellen, befindet sich im Mittelfeld. 
Tab. 2: Relative Risiken der Sterblichkeit für Lebendgeborene in Rostock-St. Jakobi 1815-1829 (Risikopopulation); Ereignis: Tod im 1. Lebensjahr; Prozesszeit: Alter in Tagen

\begin{tabular}{|c|c|c|c|c|}
\hline \multirow{2}{*}{$\begin{array}{l}\text { Variablen und } \\
\text { Ausprägungen }\end{array}$} & \multicolumn{2}{|c|}{ Modell 1: Beruf } & \multicolumn{2}{|c|}{ Modell 2: Legitimität } \\
\hline & $\begin{array}{l}\text { nur Beruf } \\
\text { des Vaters }\end{array}$ & $\begin{array}{l}\text { mit Kontroll- } \\
\text { variablen }\end{array}$ & nur Legitimität & $\begin{array}{c}\text { mit Kontroll- } \\
\text { variablen }\end{array}$ \\
\hline \multicolumn{5}{|l|}{ Beruf des Vaters } \\
\hline Gehobene Tätigkeit (RG) & 1 & 1 & & \\
\hline Mittlere Tätigkeit & $1,35^{*}$ & $1,34^{*}$ & & \\
\hline Einfache Tätigkeit & $1,55^{* *}$ & $1,55^{* *}$ & & \\
\hline Sonstige, unbekannt & 0,97 & 0,97 & & \\
\hline \multicolumn{5}{|l|}{ Legitimität der Geburt } \\
\hline Ehelich (RG) & & & 1 & 1 \\
\hline Unehelich & & & 0,79 & 0,79 \\
\hline \multicolumn{5}{|l|}{ Geschlecht } \\
\hline Weiblich (RG) & & 1 & & 1 \\
\hline Männlich & & 1,00 & & 1,00 \\
\hline \multicolumn{5}{|l|}{ Saisonalität bei Geburt } \\
\hline Frühling (RG) & & 1 & & 1 \\
\hline Sommer & & 0,97 & & 0,98 \\
\hline Herbst & & 1,01 & & 1,02 \\
\hline Winter & & 0,92 & & 0,92 \\
\hline Fallzahl (N) & 2768 & 2768 & 2768 & 2768 \\
\hline Ereignisse $(\mathrm{E})$ & 331 & 331 & 331 & 331 \\
\hline Risikozeit (t) in Tagen & 923404 & 923404 & 923404 & 923404 \\
\hline
\end{tabular}

Für die unehelichen Lebendgeburten ist das relative Risiko, das erste Lebensjahr nicht zu überleben, im Vergleich zur Referenzgruppe der Ehelichgeborenen um $21 \%$ geringer. Dieser Unterschied ist jedoch nicht signifikant. Im Hinblick auf die Kontrollvariablen Geschlecht und Saisonalität sind die Unterschiede zwischen den Ausprägungen minimal und nicht signifikant.

Im Folgenden wird das Cox-Modell noch einmal getrennt für die ersten 30 Lebenstage und für die verbleibende Zeit vom zweiten bis zum zwölften Lebensmonat dargestellt (Tab. 3 und 4).

Beide Tabellen zeigen bei den Berufsgruppen erhöhte Risiken für die mittlere und niedrige Berufsschicht. Dieser Befund ist im 1. Lebensmonat (Tab. 3) jedoch viel stärker ausgeprägt und signifikant. Für die mittlere Schicht ist der Hazard Ratio 
Tab. 3: Relative Risiken der Sterblichkeit für Lebendgeborene in Rostock-St. Jakobi 1815-1829 (Risikopopulation); Ereignis: Tod im 1. Lebensmonat; Prozesszeit: Alter in Tagen

\begin{tabular}{|c|c|c|c|c|}
\hline \multirow{2}{*}{$\begin{array}{l}\text { Variablen und } \\
\text { Ausprägungen }\end{array}$} & \multicolumn{2}{|c|}{ Modell 1: Beruf } & \multicolumn{2}{|c|}{ Modell 2: Legitimität } \\
\hline & $\begin{array}{l}\text { nur Beruf } \\
\text { des Vaters }\end{array}$ & $\begin{array}{l}\text { mit Kontroll- } \\
\text { variablen }\end{array}$ & nur Legitimität & $\begin{array}{l}\text { mit Kontroll- } \\
\text { variablen }\end{array}$ \\
\hline \multicolumn{5}{|l|}{ Beruf des Vaters } \\
\hline Gehobene Tätigkeit (RG) & 1 & 1 & & \\
\hline Mittlere Tätigkeit & $1,62^{*}$ & $1,65^{*}$ & & \\
\hline Einfache Tätigkeit & $1,92^{* *}$ & $1,89 * *$ & & \\
\hline Sonstige, unbekannt & 1,04 & 1,06 & & \\
\hline \multicolumn{5}{|l|}{ Legitimität der Geburt } \\
\hline Ehelich (RG) & & & 1 & 1 \\
\hline Unehelich & & & 0,68 & 0,69 \\
\hline \multicolumn{5}{|l|}{ Geschlecht } \\
\hline Weiblich (RG) & & 1 & & 1 \\
\hline Männlich & & 1,13 & & 1,12 \\
\hline \multicolumn{5}{|l|}{ Saisonalität bei Geburt } \\
\hline Frühling (RG) & & 1 & & 1 \\
\hline Sommer & & $1,56^{*}$ & & $1,56^{*}$ \\
\hline Herbst & & 1,43 & & 1,44 \\
\hline Winter & & 1,21 & & 1,20 \\
\hline Fallzahl (N) & 2768 & 2768 & 2768 & 2768 \\
\hline Ereignisse (E) & 120 & 120 & 120 & 120 \\
\hline Risikozeit (t) in Tagen & 923404 & 923404 & 923404 & 923404 \\
\hline \multicolumn{5}{|c|}{$\begin{array}{l}{ }^{*} \mathrm{p} \leq 0,1 ;{ }^{* *} \mathrm{p} \leq 0,05 ;{ }^{* *} \mathrm{p} \leq 0,01 . \\
\mathrm{RG}=\text { Referenzgruppe }\end{array}$} \\
\hline \multicolumn{5}{|c|}{$\begin{array}{l}\text { Quelle: Beerdigungs- und Taufregister von St. Jakobi, Rostock (eigene Berechnungen) } \\
\text { um } 62 \% \text { im Vergleich zur Referenzgruppe erhöht, für die niedrige Schicht sind es } \\
92 \% \text {. Die Restkategorie unterscheidet sich in beiden Perioden nicht von der Refe- } \\
\text { renzgruppe. } \\
\text { Hinsichtlich der Legtimität ist der Hazard Ratio für die unehelich geborenen } \\
\text { Säuglinge in den ersten } 30 \text { Lebenstagen um } 31 \% \text {, im Folgezeitraum um } 15 \% \text { im } \\
\text { Vergleich zu den ehelich geborenen geringer. Dieser Unterschied ist aber nicht sig- } \\
\text { nifkant. Auch die Geschlechterunterschiede sind nicht signifikant. Es fällt aber auf, } \\
\text { dass sich der Hazard Ratio im ersten Zeitraum ein wenig zu Gunsten der Mädchen, } \\
\text { im zweiten Zeitraum zu Gunsten der Jungen darstellt. Die saisonalen Differenzen } \\
\text { sind in den beiden Teilzeiträumen stärker ausgeprägt als im gesamten ersten Le- } \\
\text { bensjahr. So ist für die ersten } 30 \text { Lebenstage das relative Risiko für im Sommer Ge- } \\
\text { borene signifikant um } 56 \% \text { (Tab. 3, Modell 2) im Vergleich zu den im Frühling Gebo- } \\
\text { renen erhöht, während die Sommergeburten im Folgezeitraum das vergleichsweise } \\
\text { geringste Risiko aufweisen. }\end{array}$} \\
\hline
\end{tabular}


Tab. 4: $\quad$ Relative Risiken der Sterblichkeit für Lebendgeborene in RostockSt. Jakobi 1815-1829 (Risikopopulation); Ereignis: Tod im 2.-12. Lebensmonat; Prozesszeit: Alter in Tagen

\begin{tabular}{|c|c|c|c|c|}
\hline \multirow{2}{*}{$\begin{array}{l}\text { Variablen und } \\
\text { Ausprägungen }\end{array}$} & \multicolumn{2}{|c|}{ Modell 1: Beruf } & \multicolumn{2}{|c|}{ Modell 2: Legitimität } \\
\hline & $\begin{array}{c}\text { nur Beruf } \\
\text { des Vaters }\end{array}$ & $\begin{array}{l}\text { mit Kontroll- } \\
\text { variablen }\end{array}$ & nur Legitimität & $\begin{array}{l}\text { mit Kontroll- } \\
\text { variablen }\end{array}$ \\
\hline \multicolumn{5}{|l|}{ Beruf des Vaters } \\
\hline Gehobene Tätigkeit (RG) & 1 & 1 & & \\
\hline Mittlere Tätigkeit & 1,22 & 1,20 & & \\
\hline Einfache Tätigkeit & 1,38 & 1,39 & & \\
\hline Sonstige, unbekannt & 0,93 & 0,92 & & \\
\hline \multicolumn{5}{|l|}{ Legitimität der Geburt } \\
\hline Ehelich (RG) & & & 1 & 1 \\
\hline Unehelich & & & 0,85 & 0,85 \\
\hline \multicolumn{5}{|l|}{ Geschlecht } \\
\hline Weiblich (RG) & & 1 & & 1 \\
\hline Männlich & & 0,93 & & 0,93 \\
\hline \multicolumn{5}{|l|}{ Saisonalität bei Geburt } \\
\hline Frühling (RG) & & 1 & & 1 \\
\hline Sommer & & 0,75 & & 0,76 \\
\hline Herbst & & 0,85 & & 0,86 \\
\hline Winter & & 0,82 & & 0,82 \\
\hline Fallzahl (N) & 2768 & 2768 & 2768 & 2768 \\
\hline Ereignisse (E) & 211 & 211 & 211 & 211 \\
\hline Risikozeit (t) in Tagen & 923404 & 923404 & 923404 & 923404 \\
\hline
\end{tabular}

${ }^{*} \mathrm{p} \leq 0,1 ;{ }^{*} \mathrm{p} \leq 0,05 ;{ }^{* *} \mathrm{p} \leq 0,01$.

$\mathrm{RG}=$ Referenzgruppe

Quelle: Beerdigungs- und Taufregister von St. Jakobi, Rostock (eigene Berechnungen)

\section{Diskussion}

In St. Jakobi, einem der vier Kirchspiele der Stadt Rostock, hängt die Säuglingssterblichkeit zu Beginn des 19. Jahrhunderts signifikant von der beruflichen Tätigkeit des Vaters ab. Dieser Zusammenhang ist besonders stark in den ersten dreißig Lebenstagen ausgeprägt, bleibt aber auch tendenziell in den folgenden elf Monaten erhalten. Kinder von beruflich schlechter gestellten Vätern wie niedrigere Verwaltungsangestellte, Seefahrer, Fischer und Ackersmänner, haben das höchste Risiko, im ersten Lebensjahr zu versterben, jene der Gruppe der höheren Beamten, Händler und Juristen, die die Elite Rostocks bildeten (Manke 2000: 369-370), haben das niedrigste Sterberisiko. Entsprechend der im 2. Abschnitt aufgeführten Literatur können diese Unterschiede mit der wirtschaftlichen Situation der Familie, der Ernährung der Mutter während der Schwangerschaft aber auch des Neugeborenen 
in den ersten Lebensmonaten zusammenhängen. Hinzu kommt, dass in der Landwirtschaft auch die Mütter stärker in die Arbeit involviert sind und somit weniger Zeit für die Nachkommen haben. Auch die Kinder von Arbeitern aus der Seefahrt weisen ein vergleichsweise hohes Risiko auf, das durch geringe Einkünfte und lange und häufige Abwesenheit der Väter erklärt werden kann.

Ungewöhnlich ist das Ergebnis zur Legitimität der Geburten. In der Literatur wird für uneheliche Geburten sowohl für Rostock als auch für ganz Deutschland stets eine erhöhte Säuglingssterblichkeit nachgewiesen, zumindest im späten 19. und frühen 20. Jahrhundert (z.B. Brüning/Balck 1906 und Brüning/Josephy 1928 für Rostock sowie Prinzing 1902 und 1911 für das Deutsche Reich). Dieser Effekt ist jedoch in St. Jakobi nicht auszumachen, tendenziell haben unehelich Geborene sogar höhere Überlebenschancen, auch wenn der Effekt statistisch nicht signifikant ist. Möglicherweise handelte es sich bei den unverheirateten Müttern zum Teil um Dienstboten, die ihre Kinder nach der Taufe den auf dem Land lebenden Großmüttern oder anderen Vertrauten oder einer Institution übergaben oder vielleicht auch selbst auf das Land zurückgingen. Vögele (1994) sowie Preston und Haines (1991) begründen dieses Phänomen mit der ungünstigen finanziellen Situation, welche alleinstehende Mütter zwang, weiter arbeiten zu gehen und ihr Kind an Verwandte oder eine Einrichtung abzugeben. Auf diese Weise wären Säuglingssterbefälle nicht in St. Jakobi verzeichnet worden. Anlass zu dieser Spekulation liefert auch der hohe Anteil von Dienstboten an der weiblichen Bevölkerung Rostocks. Dieser betrug im Jahr 1800 immerhin $30 \%$ in der Altersgruppe 21 bis 30 (Manke 2000: 336). Wenn man zudem das Cox-Modell nur für Totgeburten rechnet, weisen unehelich Geborene ein um $28 \%$ erhöhtes Sterberisiko gegenüber den ehelich Geborene auf. Wenngleich der Unterschied auch hier nicht signifikant ist, ist dies dennoch ein weiteres Indiz für eine abwanderungsbedingte Untererfassung unehelicher Säuglingssterbefälle.

Die Jahreszeit der Geburt hat einen signifikanten Einfluss auf das Überleben in den ersten dreißig Tagen, wobei im Juni, Juli und August Geborene das höchste Sterberisiko aufweisen. Breschi und Bacci (1997) zeigen für fünf Länder, dass im 19. Jahrhundert die Sterblichkeit in den ersten beiden Lebensjahren vom Geburtsmonat abhängt und sich das Muster in den einzelnen Ländern unterscheidet. So hatten z.B. in Italien die im Sommer Geborenen eine niedrigere Sterblichkeit, in der Schweiz die im Winter Geborenen. Ein der Schweiz und St. Jakobi vergleichbares Muster findet sich für das erste Lebensjahr auch im Dänemark des 19. Jahrhunderts (Dob/hammer/Vaupel 2001; Doblhammer 2004). Da in St. Jakobi der Effekt des Geburtsmonats vor allem in den ersten dreißig Lebenstagen von Bedeutung ist, weist dieser auf endogene Faktoren der Säuglingssterblichkeit hin, die eher mit der Entwicklung des Fötus während der Schwangerschaft zusammenhängen als mit exogenen Faktoren wie Infektionserkrankungen. Da im Norden Deutschlands die Stillbereitschaft stärker ausgeprägt war als im Süden, sind in St. Jakobi die Neugeborenen durch die protektiven Substanzen der Muttermilch besser vor saisonalen Infektionserkrankungen des Magen-Darmtraktes, aber auch der Atemwege geschützt. 
Bezüglich des Geschlechts lassen sich keine signifikanten Unterschiede feststellen, auch wenn Jungen in den ersten 30 Lebenstagen, wenn endogene Faktoren, speziell das Geburtsgewicht und die allgemeine körperliche Verfassung bei Geburt, eine entscheidende Rolle spielen, ein erhöhtes Sterberisiko haben. In den Folgemonaten dreht sich dieser Effekt jedoch um.

Ein Faktor, der in dieser Studie nicht berücksichtigt werden konnte, ist die Taufe. Grundsätzlich ist es eine Stärke des Datensatzes, dass darin sowohl Informationen über das Tauf- als auch über das Geburtsdatum enthalten sind. Es sind auch ungetaufte Fälle im Modellzeitraum 1815 bis 1829 zu verzeichnen, wobei es sich dabei ausschließlich um Totgeburten handelt. Juden sind beispielsweise nicht in den Registern enthalten. Während die Zeitspanne von der Geburt bis zur Taufe im Untersuchungszeitraum stets nur wenige Tage beträgt, wird sie zum Ende des 19. Jahrhunderts immer größer, bis hin zu einigen Wochen oder gar Monaten, wie spätere Jahrgänge der Kirchenbücher von St. Jakobi zeigen. Jedoch gilt im frühen 19. Jahrhundert ebenso wie im späteren: Wenn abzusehen war, dass ein Kind nach der Geburt bald sterben würde, wurde die Nottaufe vorgenommen, in der Regel durch die Hebamme, wie Anmerkungen in den Tauf- und Beerdigungsregistern deutlich machen.

Fraglich ist, ob es sich bei den registrierten Totgeburten tatsächlich um solche handelt. Im 19. Jahrhundert gab es noch keine festen Definitionen für Lebend- und Totgeburt. Ob ein toter Säugling zuvor Lebenszeichen hatte, war vom Pfarrer zu entscheiden. Die Kriterien hierfür sind jedoch nicht bekannt. Ein Zusammenhang mit der Taufe scheint bei Betrachtung des vorliegenden Datensatzes im Zeitraum 1815 bis 1829 nicht ausgeschlossen. Möglich wäre, dass ein Neugeborenes erst ab der Taufe als Lebendgeburt betrachtet wurde und es, wenn es ungetauft verstarb, als Totgeburt verzeichnet wurde. Dies könnte mit anderen Rostocker Kirchenbüchern aus der gleichen Zeit überprüft werden. Für die zweite Hälfte des 19. Jahrhunderts ist dies als gängige Praxis der Abgrenzung jedoch nicht belegbar, zumindest nicht für St. Jacobi, da in dieser Zeit auch ungetauft verstorbene Lebendgeburten in den Registern enthalten sind.

Zusammenfassend kann festgehalten werden, dass die vorliegenden Individualdaten von St. Jakobi neue Einblicke in die Säuglingssterblichkeit einer mehrheitlich städtischen Bevölkerung Norddeutschlands im frühen 19. Jahrhundert bieten. Damit wird eine Forschungslücke geschlossen. Für weitere Analysen wäre eine Ausweitung des Untersuchungszeitraumes notwendig, einerseits um größere Fallzahlen zu generieren, andererseits um die zeitliche Entwicklung der sozialen Unterschiede in der Säuglingssterblichkeit betrachten zukönnen. Letzteres ist von besonderem Interesse, da etwa ab 1840 die Säuglingssterblichkeit wieder ansteigt und es offen ist, inwieweit alle sozialen Gruppen von diesem Anstieg betroffen sind.

Weitere Studienmöglichkeiten würden sich durch die Verknüpfung der Daten von St. Jakobi mit den Rostocker Volkszählungen des 19. Jahrhunderts und den Kopulations- und Konfirmationsregistern von St. Jakobi ergeben. Zusätzliche Faktoren der Säuglingssterblichkeit, unter anderem auf der Haushaltsebene, könnten so in die Analyse mit einfließen (Scholz 2013). Man erhielte mehr Informationen über die Eltern, wie das Geburtsdatum der Mutter und des Vaters, welches ab 1853 in 
den Rostocker Kopulationsregistern erfasst wurde. Zudem finden sich Informationen über Geburtenabstände, Geburtsränge und Geschwisterzahl. Damit wären die Daten nicht nur für die weitere Mortalitätsforschung interessant, sondern auch für Fertilitäts- und Sozialstrukturanalysen sowie für genealogische Studien.

\section{Literatur}

Allison, Paul D. 1984: Event History Analysis. Regression for Longitudinal Event Data. Beverly Hills: Sage Publications.

Ariés, Philippe 1975: Geschichte der Kindheit. München: Carl Hanser Verlag.

Backhaus, Klaus; Erichson, Bernd; Plinke, Wulff; Weiber, Rolf 2006: Multivariate Analysemethoden. Eine anwendungsorientierte Einführung. 11. Auflage. Berlin: Springer [doi: 10.1007/3-540-29932-7].

Becker, Karl M. L. 1874: Zur Berechnung von Sterbetafeln an die Bevölkerungsstatistik zu stellende Anforderungen. Berlin: Verlag des Königlichen Statistischen Bureaus.

Bengtsson, Tommy; Lundh, Christer 1994: La mortalité infantile et post-infantile dans les pays Nordiques avant 1900. In: Annales de Démographie historique 1994: 23-43.

Blayo, Yves 1975: La mortalité en France de 1740 à 1829. In: Population 30, Sonderband "Démographie historique": 123-142.

Blossfeld, Hans-Peter; Hamerle, Alfred; Mayer, Karl-Ulrich 1986: Ereignisanalyse. Frankfurt: Campus.

Bluhm, Agnes 1912: Stillhäufigkeit und Stilldauer. In: Grotjahn, Alfred; Kaup, Ignaz (Hrsg.): Handwörterbuch der Sozialen Hygiene. Band 2. Leipzig: F. C. W. Vogel: 570 591.

Brandenburg, Hajo et al. 1991: Berufe in Altona 1803. Berufssystematik für eine präindustrielle Stadtgesellschaft anhand der Volkszählung. Kleine Schriften des Arbeitskreises für Wirtschafts- und Sozialgeschichte Schleswig-Holsteins, Band 1. Kiel: Arbeitskreis für Wirtschafts- und Sozialgeschichte Schleswig-Holsteins.

Brandenburg, Hajo; Kroll, Stefan 1998: „Erwerbsstruktur" und „Beruf“. Möglichkeiten und Grenzen einer Berufssystematik für präindustrielle Stadtgesellschaften. In: Krüger, Kersten; Kroll, Stefan (Hrsg.): Die Sozialstruktur der Städte Kiel und Altona um 1800. Demographie, Erwerbsstruktur und wirtschaftliche Leistungsfähigkeit. Studien zur Wirtschafts- und Sozialgeschichte Schleswig-Holsteins, Band 29. Neumünster: Wachholtz Verlag: 21-29.

Breschi, Marco; Bacci, Massimo Livi 1997: Month of Birth as a Factor in Children's Survival. In: Bideau, Alain; Desjardins, Bertrand; Brignoli, Héctor Pérez (Hrsg.): Infant and Child Mortality in the Past. Oxford: Clarendon Press: 157-173.

Breschi, Marco et al. 2014: Social and Economic Determinants of Reproductive Behavior before the Fertility Decline. The Case of Six Italian Communities during the Nineteenth Century. In: European Journal of Population 30,3: 291-315 [doi: 10.1007/s10680-0139303-8].

Brüning, Hermann 1908: Säuglingssterblichkeit im Großherzogtum MecklenburgSchwerin in den Jahren 1876-1905. Nach einem Vortrag. Aus der medizinischen Kinderabteilung des Universitäts-Krankenhauses zu Rostock. In: Zeitschrift für Säuglingsfürsorge 2: 367-378. 
Brüning, Hermann 1928: Über die Frühsterblichkeit der Säuglinge in MecklenburgSchwerin in der Zeit vor und nach dem Kriege 1901-1905 und 1921-1925. In: Archiv für Kinderheilkunde 86,1: 1-8.

Brüning, Hermann; Balck, R. 1906: Säuglingssterblichkeit in Rostock. In: Zeitschrift für Säuglingsfürsorge 1: 338-355.

Brüning, Hermann; Josephy, Edith 1928: Über Säuglingssterblichkeit in Rostock. In: Gesundheitsfürsorge für das Kindesalter 3,2-3: 141-152.

Brüning, Hermann; Mahlo, Erwin 1929: Ein weiterer Beitrag zur Frühsterblichkeit der mecklenburgischen Säuglinge vor und nach dem Kriege. In: Archiv für Kinderheilkunde 86,2: 122-140.

Brüning, Hermann; Stein, Ernst W. M. 1911: Die Säuglingssterblichkeit im Großherzogtum Mecklenburg-Strelitz. Nebst Bemerkungen über ihre Ursachen und Bekämpfung. In: Zeitschrift für Säuglingsschutz 3: 142-222.

Diekmann, Andreas; Mitter, Peter 1984: Methoden zur Analyse von Zeitverläufen. Stuttgart: Teubner.

Dinkel, Reiner H. 1984: Sterblichkeit in Perioden- und Kohortenbetrachtung. In: Zeitschrift für Bevölkerungswissenschaft 10,4: 477-500.

Dippe, Martin C. 1857: Die lange Dauer des Lebens in Mecklenburg. In: Archiv für Landeskunde in den Grossherzogtümern Mecklenburg 8: 545-562.

Doblhammer, Gabriele; Vaupel, James W. 2001: Life Span Depends on Month of Birth. In: Proceedings of the National Academy Sciences of the United States of America 98,5: 2934-2939 [doi: 10.1073/pnas.041431898].

Doblhammer, Gabriele 2004: The Late Life Legacy of Very Early Life. Demographic Research Monographs. Berlin/Heidelberg: Springer Verlag [http://www.demogr.mpg.de/ books/drm/002/, 24.02.2015] [doi: 10.1007/978-3-662-10349-4].

Ehling, Manfred 1996: Historische Statistik. Perspektiven der internationalen Zusammenarbeit. In: Wirtschaft und Statistik 7: 413-421.

Ehmer, Josef 2004: Bevölkerungsgeschichte und historische Demographie 1800-2000. München: Oldenbourg.

Esenwein-Rothe, Ingeborg 1982: Einführung in die Demographie. Bevölkerungsstruktur und Bevölkerungsprozess aus der Sicht der Statistik. Wiesbaden: Franz Steiner Verlag.

Gehrmann, Rolf 2000: Bevölkerungsgeschichte Norddeutschlands zwischen Aufklärung und Vormärz. Schriftenreihe des Forschungsinstituts für die Geschichte Preußens e.V., Band 1. Berlin: Berlin Verlag Arno Spitz.

Gehrmann, Rolf 2002: Infant Mortality in Town and Countryside: Northern Germany, ca. 1750-1850. In: The History of the Family 7: 545-556.

Gehrmann, Rolf 2011: Säuglingssterblichkeit in Deutschland im 19. Jahrhundert. In: Comparative Population Studies - Zeitschrift für Bevölkerungswissenschaft 36,4: 807838 [doi: 10.4232/10.CPoS-2011-22de].

Haines, Michae/ R. 1995: Socio-economic Differentials in Infant and Child Mortality during Mortality Decline: England and Wales, 1890-1911. In: Population Studies 49,2: 297-315 [doi: 10.1080/0032472031000148526].

Halley, Edmond 1693: An Estimate of the Degrees of the Mortality of Mankind, drawn from curious Tables of the Births and Funerals at the City of Breslaw, with an Attempt to ascertain the Price of Annuities upon Lives. In: Philosophical Transactions 196: 596-610.

Hanssen, Peter 1912: Die Säuglingssterblichkeit der Provinz Schleswig-Holstein und die Mittel zu ihrer Abhilfe. Kiel: Handorff. 
Heller, Geneviève; Imhof, Arthur E. 1983: Körperliche Überlastung der Frauen im 19. Jahrhundert. In: Imhof, Arthur E. (Hrsg.): Leib und Leben in der Geschichte der Neuzeit. Berlin: Duncker \& Humblot.

Höfler, Max 1899: Deutsches Krankheitsnamen-Buch. München: Piloty \& Loehle. Nachdruck: Hildesheim: Georg Olms (1970).

Hollingsworth, Thomas H. 1969: Historical Demography. Ithaca/New York: Cornell University Press.

Hosmer, David W.; Lemeshow, Stanley 2000: Applied Logistic Regression. Second Edition. New York: John Wiley \& Sons.

Imhof, Arthur E. 1977: Einführung in die historische Demographie. München: C. H. Beck.

Imhof, Arthur E. 1981: Unterschiedliche Säuglingssterblichkeit in Deutschland, 18. bis 20. Jahrhundert - Warum? In: Zeitschrift für Bevölkerungswissenschaft 7,3: 343-382.

Johansson, Kent 2004: Child Mortality during the Demographic Transition. A Longitudinal Analysis of a Rural Population in Southern Sweden, 1766-1894. Stockholm: Almquist \& Wiksell International.

Johansson, Kent 2009: Interaction Effects of Infection and Malnutrition on Child Mortality in Scania, Sweden 1766-1894. Conference paper presented at the IUSSP meeting in Marrakech, Maroc 28 September - 2 October 2009.

Kintner, Hallie J. 1985: Trends and Regional Differences in Breastfeeding in Germany from 1871 to 1937. In: Journal of Family History 10: 163-182 [doi: 10.1177/036319908501000203].

Kintner, Hallie J. 1994: Infant Mortality Decline in Germany, 1871-1925. The Roles of Changes in Variables and Changes in the Structure of Relations. In: Genus 50,3-4: 117-132.

Kloke, Ines E. 1997: Säuglingssterblichkeit in Deutschland im 18. und 19. Jh. am Beispiel von sechs ländlichen Regionen. Berlin. Digitale Dissertation [https://www.phf. uni-rostock.de/tthist/kloke/iekloke.pdf, 25.11.2013].

Knodel, John E. 1968: Infant Mortality and Fertility in Three Bavarian Villages. An Analysis of Family Histories from the 19th Century. In: Population Studies 22: 297-318 [doi: 10.1080/00324728.1968.10404941].

Knodel, John E. 1988: Demographic Behaviour in the Past. A Study of Fourteen German Village Populations in the Eighteenth and Nineteenth Centuries. New York: Cambridge University Press.

Kölbl, Stefanie 2004: Das Kinderdefizit im frühen Mittelalter. Realität oder Hypothese? Zur Deutung demographischer Strukturen in Gräberfeldern. Tübingen. Digitale Dissertation [http://tobias-lib.uni-tuebingen.de/volltexte/2004/1152/pdf/diss_a4.pdf, 25.11.2013].

Köppe, Tobias 2010: Kirchensprengung und -abriss in der Deutschen Demokratischen Republik. Rostock [http://www.kirchensprengung.de/cms/kirchensprengung rostock.html, 25.11.2013].

Krieg, Georg 1890: Bewegung der Bevölkerung im Königreich Bayern im Jahresdurchschnitt der Periode 1879/88. Mit einleitenden Bemerkungen und Rückblicken auf die vier Jahrzehnte 1838/78. In: Königlich statistisches Bureau (Hrsg.): Beiträge zur Statistik des Königreichs Bayern LVI. München: Lindauer.

Krüger, Kersten; Kroll, Stefan (Hrsg.) 1998: Die Sozialstruktur der Städte Kiel und Altona um 1800. Demographie, Erwerbsstruktur und wirtschaftliche Leistungsfähigkeit. Studien zur Wirtschafts- und Sozialgeschichte Schleswig-Holsteins, Band 29. Neumünster: Wachholtz. 
Krüger, Kersten 1998: Norddeutsche Hafenstädte um 1800: Altona, Kiel, Rostock und Wismar. Ein sozialgeschichtlicher Vergleich. In: Historical Social Research 23,3: 90 128.

Krüger, Kersten 2000: Das Gewerbe in Altona und Kiel 1803, Rostock und Wismar 1819. In: Kaufhold, Karl H.; Reininghaus, Wilfried (Hrsg.): Stadt und Handwerk in Mittelalter und früher Neuzeit. Köln/Weimar/Wien: Böhlau: 159-168.

Krüger, Kersten 2003: Erwerbsleben in Altona, Kiel, Rostock und Wismar an der Schwelle zur Moderne um 1800. In: Krüger, Kersten; Pápay, Gyula; Kroll, Stefan (Hrsg.): Stadtgeschichte und Historische Informationssysteme. Der Ostseeraum im 17. und 18. Jahrhundert. Beiträge des wissenschaftlichen Kolloquiums in Rostock vom 21. und 22. März 2002. Münster: Lit Verlag: 247-271.

Kundmann, Johann C. 1737: Reflexiones über die Kranckheits- und Todten-Listen, mit Medicinischen Anmerckungen begleitet. In: Kundmann, Johann C. (Hrsg.): Rariora Naturæ \& Artis item in Re Medica, oder Seltenheiten der Natur und Kunst des Kundmannischen Naturalien-Cabinets, wie auch in der Artzeney-Wissenschaft. Breslau/Leipzig: Ben Michael Hubert: 1248-1312.

Kuzia, Detlef 2004: Historische Rostocker Bauwerke. Rostocker Jakobi-Kirche. Reich und doch nicht erste Adresse [http://www.mv-terra-incognita.de/beitraege/denkmale/verlorene/jakobi/jakobi1.htm, 25.11.2013].

Lee, Robert; Marschalck, Peter 2002: Infant Mortality in Bremen in the 19th Century. In: The History of the Family 7: 557-583 [doi: 10.1016/S1081-602X(02)00127-6].

Lorenzen-Schmidt, Klaus-Joachim 1996: Lexikon historischer Berufe in Schleswig-Holstein und Hamburg. Kleine Schriften des Arbeitskreises für Wirtschafts- und Sozialgeschichte Schleswig-Holsteins, Band 2. Kiel: Arbeitskreis für Wirtschafts- und Sozialgeschichte Schleswig-Holsteins.

Manke, Matthias 2000: Rostock zwischen Revolution und Biedermeier. Alltag und Sozialstruktur. Rostocker Studien zur Regionalgeschichte, Band 1. Rostock: Ingo Koch Verlag.

Manke, Matthias 2005a: Einleitung. Die mecklenburgische Volkszählung von 1819. In: Manke, Matthias (Hrsg.): „... dass alle Welt geschätzt würde.“ Die Einwohner der Stadt Rostock nach der Volkszählung von 1819. Rostock: Ingo Koch Verlag: 7-26.

Manke, Matthias 2005b: Die Volkszählungen des Großherzogtums Mecklenburg-Schwerin in den Jahren 1819 und 1867 unter Berücksichtigung der Volkszählung des Jahres 1866. In: Genealogie 54,3-4: 449-468.

Medick, Hans 1997: Weben und Überleben in Laichingen 1650-1900. Lokalgeschichte als Allgemeine Geschichte. Veröffentlichungen des Max-Planck-Instituts für Geschichte, Band 126. Göttingen: Vandenhoeck \& Ruprecht.

Mitchell, Brian R. 2007: International Historical Statistics. Europe 1750-2005. Sixth Edition. Basingstoke/New York: Palgrave Macmillan.

Mühlichen, Michae/ 2011: Säuglingssterblichkeit in Rostock im 19. Jahrhundert. Eine demografische Analyse anhand von Kirchenbuchdaten. Masterarbeit an der Wirtschaftsund Sozialwissenschaftlichen Fakultät der Universität Rostock.

Mühlichen, Michael; Scholz, Rembrandt D. 2015: Dokumentation der Aufbereitung historischer Kirchenbuchdaten am Beispiel der Säuglingssterblichkeit in der Hansestadt Rostock. BiB Daten- und Methodenberichte 2/2015. Wiesbaden: Bundesinstitut für Bevölkerungsforschung.

North Atlantic Population Project (NAPP) 2013: OCCHISCO. Occupation, HISCO Classification [https://www.nappdata.org/napp-action/variables/OCCHISCO\#codes_section, 25.11.2013]. 
Paulsen, Christian 1909: Die Säuglingssterblichkeit in den mecklenburgischen Aushebungsbezirken in den Jahren 1876-1905. Inaugural-Dissertation zur Erlangung der Doktorwürde der medizinischen Fakultät der Universität zu Rostock. Rostock: Adler.

Preston, Samuel H.; Haines, Michael R. 1991: Fatal Years. Child Mortality in Late Nineteenth-Century America. Princeton: Princeton University Press.

Prinzing, Friedrich 1899: Die Entwicklung der Kindersterblichkeit in den europäischen Staaten. In: Jahrbücher für Nationalökonomie und Statistik 72: 577-635.

Prinzing, Friedrich 1900: Die Kindersterblichkeit in Stadt und Land. In: Jahrbücher für Nationalökonomie und Statistik 75: 593-645.

Prinzing, Friedrich 1902: Die uneheliche Fruchtbarkeit in Deutschland. In: Zeitschrift für Sozialwissenschaft 5: 37-46.

Prinzing, Friedrich 1911: Uneheliche Geburten. In: Conrad, Johannes (Hrsg.): Handwörterbuch der Staatswissenschaften. Jena: Fischer: 34-43.

Prinzing, Friedrich 1931: Handbuch der medizinischen Statistik. Zweite, vollständig umgearbeitete Auflage. Jena: Fischer.

Rödel, Walter G. 1990: "Statistik“ in vorstatistischer Zeit. Möglichkeiten und Probleme der Erforschung frühneuzeitlicher Populationen. In: Andermann, Kurt; Ehmer, Hermann (Hrsg.): Bevölkerungsstatistik an der Wende vom Mittelalter zur Neuzeit. Quellen und methodische Probleme im überregionalen Vergleich. Sigmaringen: Thorbecke: 9-25.

Saul, Rudolf 1909: Säuglingssterblichkeit im Großherzogtum Mecklenburg-Schwerin. Inaugural-Dissertation zur Erlangung der Doktorwürde der medizinischen Fakultät der Universität zu Rostock. Rostock: Adler.

Scholz, Rembrandt D. 2013: Zur demographischen Entwicklung der Hansestadt Rostock im 19. Jahrhundert. In: Busch, Michael; Kroll, Stefan; Scholz, Rembrandt D. (Hrsg.): Geschichte - Kartographie - Demographie. Historisch-Geographische Informationssysteme im methodischen Vergleich. Geschichte: Forschung und Wissenschaft, Band 45. Münster: LIT Verlag: 195-227.

Schultz, Helga 1991: Social Differences in Mortality in the Eighteenth Century. An AnaIysis of Berlin Church Registers. In: International Review of Social History 2: 232-248.

Schulz, Inga-Marie 2008: Die Säuglingssterblichkeit in Rostock im Zeitraum 1875-1910. Eine Studie auf Basis der Sterberegister der Kirchengemeinde St. Jakobi. Bachelorarbeit an der Wirtschafts- und Sozialwissenschaftlichen Fakultät der Universität Rostock.

Spree, Reinhard 1980: Die Entwicklung der differentiellen Säuglingssterblichkeit in Deutschland seit der Mitte des 19. Jahrhunderts. Ein Versuch zur Mentalitätsgeschichte. In: Imhof, Arthur E. (Hrsg.): Mensch und Gesundheit in der Geschichte. Husum: Matthiessen: 251-278 [doi: 10.1017/S0025727300036115].

Spree, Reinhard 1988: Volksgesundheit und Lebensbedingungen in Deutschland während des frühen 19. Jahrhunderts. In: Jahrbuch des Instituts für Geschichte der Medizin der Robert Bosch Stiftung 7: 75-113.

Spree, Reinhard 1998: Der Rückzug des Todes. Der epidemiologische Übergang in Deutschland während des 19. und 20. Jahrhunderts. In: Historical Social Research 23,1-2: 4-43.

Stöckel, Sigrid 1986: Säuglingssterblichkeit in Berlin von 1870 bis zum Vorabend des Ersten Weltkriegs. Eine Kurve mit hohem Maximum und starkem Gefälle. In: Ribbe, Wolfgang (Hrsg.): Berlin-Forschungen, Band 1. Berlin: Colloquium: 219-264. 
Sundin, Jan 1995: Culture, Class, and Infant Mortality during the Swedish Mortality Transition, 1750-1850. In: Social Science History 19,1: 117-145.

Süßmilch, Johann Peter 1761: Die göttliche Ordnung in den Veränderungen des menschlichen Geschlechts, aus der Geburt, dem Tode und der Fortpflanzung desselben erwiesen. Zwote und ganz umgearbeitete Ausgabe. Berlin: Verlag des Buchladens der Realschule.

Szołtysek, Mikołaj et al. 2010: Living Arrangements and Household Formation in the Crucible of Social Change. Rostock 1867-1900. MPIDR Working Paper 2010-036. Rostock: Max-Planck-Institut für demografische Forschung.

Vögele, Jörg P. 1994: Urban Infant Mortality in Imperial Germany. In: Social History of Medicine 7,3: 401-425 [doi: 10.1093/shm/7.3.401].

Waldron, Ingrid 1983: Sex Differences in Human Mortality. The Role of Genetic Factors. In: Social Science and Medicine 17,6: 321-333 [doi: 10.1016/0277-9536(83)90234-4].

Westergaard, Harald 1932: Contributions to the History of Statistics. London: P. S. King and Son.

Wolleswinkel-van den Bosch, Judith H. et al. 2000: Determinants of Infant and Early Childhood Mortality Levels and Their Decline in The Netherlands in the Late Nineteenth Century. In: International Journal of Epidemiology 29,6: 1031-1040 [doi: 10.1093/ije/29.6.103].

Woods, Robert I. 1994: La mortalité infantile en Grand-Bretagne. Un bilan des connaissances historiques. In: Annales de Démographie historique 1994: 119-134.

Woods, Robert l.; Watterson, Patricia A.; Woodward, John H. 1988: The Causes of Rapid Infant Mortality Decline in England and Wales, 1861-1921 Part I. In: Population Studies 42,3: 343-366 [doi: 10.1080/0032472031000143516].

Woods, Robert l.; Watterson, Patricia A.; Woodward, John H. 1989: The Causes of Rapid Infant Mortality Decline in England and Wales, 1861-1921 Part II. In: Population Studies 43,1: 113-132 [doi: 10.1080/0032472031000143876].

Wrigley, Edward A.; Schofield, Roger S. 1981: The Population History of England 15411871. London: Edward Arnold.

Würzburg, Arthur 1887: Die Säuglingssterblichkeit im Deutschen Reiche während der Jahre 1875 bis 1877. Arbeiten aus dem Kaiserlichen Gesundheitsamte (=Beihefte zu den Veröffentlichungen aus dem Kaiserlichen Gesundheitsamte) 2: 208-222.

Würzburg, Arthur 1888: Die Säuglingssterblichkeit im Deutschen Reiche während der Jahre 1875 bis 1877. Arbeiten aus dem Kaiserlichen Gesundheitsamte (=Beihefte zu den Veröffentlichungen aus dem Kaiserlichen Gesundheitsamte) 4: 28-108.

Zeuner, Gustav A. 1869: Abhandlungen zur mathematischen Statistik. Leipzig: Arthur Felix. 
Eine Übersetzung dieses begutachteten und von den Autoren autorisierten deutschen Originaltextes durch das Bundesinstitut für Bevölkerungsforschung ist unter dem Titel "Social differences in infant mortality in 19th century Rostock. A demographic analysis based on church records", DOI 10.12765/CPoS-2015-03en bzw. URN urn:nbn:de:bib-cpos-2015-03en4, auf http://www.comparativepopulationstudies. de verfügbar.

Michael Mühlichen ( $₫$ ). Bundesinstitut für Bevölkerungsforschung. Wiesbaden, Deutschland. E-Mail: Michael.Muehlichen@bib.bund.de URL: http://www.bib-demografie.de/muehlichen

Dr. Rembrandt D. Scholz. Max-Planck-Institut für demografische Forschung. Rostock, Deutschland.E-Mail: scholz@demogr.mpg.de

URL: http://www.demogr.mpg.de/de/institut/mitarbeiter_1899/rembrandt_d_scholz_663.htm

Prof. Dr. Gabriele Doblhammer. Universität Rostock, Lehrstuhl für Empirische Sozialforschung und Demographie. Rostock, Deutschland.

E-Mail: gabriele.doblhammer@uni-rostock.de

URL: http://www.wiwi.uni-rostock.de/soziologie/esf/doblhammer/ 


\section{Comparative Population Studies}

WWW.comparativepopulationstudies.de

ISSN: 1869-8980 (Print) - 1869-8999 (Internet)

Published by / Herausgegeben von

Prof. Dr. Norbert F. Schneider

Federal Institute for Population Research

D-65180 Wiesbaden / Germany

\section{Managing Editor /}

Verantwortlicher Redakteur

Frank Swiaczny

\section{Assistant Managing Editor /}

Stellvertretende Redakteurin

Katrin Schiefer

\section{Copy Editor (German) /}

Lektorat (deutsch)

Dr. Evelyn Grünheid

\section{Layout / Satz}

Beatriz Feiler-Fuchs

E-mail:cpos@bib.bund.de

\section{Scientific Advisory Board /}

Wissenschaftlicher Beirat

Paul Gans (Mannheim)

Johannes Huinink (Bremen)

Michaela Kreyenfeld (Rostock)

Marc Luy (Wien)

Clara H. Mulder (Groningen)

Notburga Ott (Bochum)

Peter Preisendörfer (Mainz)

Zsolt Spéder (Budapest)
Board of Reviewers / Gutachterbeirat Martin Abraham (Erlangen)

Laura Bernardi (Lausanne)

Hansjörg Bucher (Bonn)

Claudia Diehl (Konstanz)

Andreas Diekmann (Zürich)

Gabriele Doblhammer-Reiter (Rostock) Jürgen Dorbritz (Wiesbaden)

Anette Eva Fasang (Berlin)

E.-Jürgen Flöthmann (Bielefeld)

Alexia Fürnkranz-Prskawetz (Wien)

Beat Fux (Salzburg)

Joshua Goldstein (Berkeley)

Karsten Hank (Köln)

Sonja Haug (Regensburg)

Hill Kulu (Liverpool)

Aart C. Liefbroer (Den Haag)

Kurt Lüscher (Konstanz)

Emma Lundholm (Umeå)

Nadja Milewski (Rostock)

Dimiter Philipov (Wien)

Roland Rau (Rostock)

Tomáš Sobotka (Wien)

Jeroen Spijker (Barcelona)

Olivier Thévenon (Paris)

Helga de Valk (Brussel)

Heike Trappe (Rostock)

Michael Wagner (Köln) 